\title{
Existence and orbital stability of standing waves to a nonlinear Schrödinger equation with inverse square potential on the half-line
}

\author{
Elek Csobo®
}

\begin{abstract}
In our work, we establish the existence of standing waves to a nonlinear Schrödinger equation with inverse-square potential on the halfline. We apply a profile decomposition argument to overcome the difficulty arising from the non-compactness of the setting. We obtain convergent minimizing sequences by comparing the problem to the problem at "infinity" (i.e., the equation without inverse square potential). Finally, we establish orbital stability/instability of the standing wave solution for mass subcritical and supercritical nonlinearities respectively.
\end{abstract}

Keywords. Nonlinear Schrödinger equation, Hardy's inequality, Standing waves, Orbital stability.

\section{Introduction}

We study the existence and orbital stability of standing waves for the following nonlinear Schrödinger equation with inverse square potential on the half line

$$
\left\{\begin{array}{l}
i u_{t}+u^{\prime \prime}+c \frac{u}{x^{2}}+|u|^{p-1} u=0 \\
u(0)=u_{0} \in H_{0}^{1}\left(\mathbb{R}^{+}\right)
\end{array}\right.
$$

where $u: \mathbb{R} \times \mathbb{R}^{+} \rightarrow \mathbb{C}, u_{0}: \mathbb{R}^{+} \rightarrow \mathbb{C}, 1<p<\infty$, and $0<c<1 / 4$.

There has been considerable interest recently in the study of the Schrödinger equation with inverse-square potential in three and higher dimensions. Classification of the so-called minimal mass blow-up solutions, global well-posedness, and stability of standing wave solutions were studied in $[1,6,8$, 22]. In the papers by Bensouilah et al. [1], and by Trachanas and Zographopoulos [22] the authors establish orbital stability of ground state solutions in the Hardy subcritical $\left(c<(N-2)^{2} / 4\right)$ and Hardy critical $\left(c=(N-2)^{2} / 4\right)$ case respectively for dimensions higher that three. In both cases, orbital stability is 
proved by showing the precompactness of minimizing sequences of the energy functional on an $L^{2}$ constraint. Local well-posedness was established for the two-dimensional space by Suzuki in [21], and in three and higher dimensions by Okazawa et al. in [18]. The presence of the inverse square potential in onedimensional space has also attracted attention. In [13] H. Kovarik and F. Truc established dispersive estimates for $\partial_{x}^{2}+c / x^{2}$.

The dynamics of the equation is closely related to Hardy's inequality (see $[7])$

$$
c \int_{0}^{\infty} \frac{|u|^{2}}{x^{2}} d x \leqslant \int_{0}^{\infty}\left|u^{\prime}\right|^{2} d x \text { for all } u \in C_{0}^{\infty}(0, \infty),
$$

where $c \leqslant 1 / 4$. We introduce the Hardy functional

$$
H(u)=\int_{0}^{\infty}\left(\left|u^{\prime}\right|^{2}-\frac{c}{x^{2}}|u|^{2}\right) d x
$$

which is closely related to our problem. We will mainly focus on the case $0<c<1 / 4$, when the natural energy space associated to $(1.1)$ is $H_{0}^{1}\left(\mathbb{R}^{+}\right)$, and the semi-norm $\left\|u^{\prime}\right\|_{L^{2}}^{2}$ is equivalent to $H(u)$.

Let us consider the operator

$$
H_{c}=-\frac{\partial^{2}}{\partial x^{2}}-\frac{c}{x^{2}}
$$

acting on $C_{0}^{\infty}\left(\mathbb{R}^{+}\right)$. Owing to the Hardy inequality, if $c<1 / 4$ the quadratic form $\left\langle H_{c} \varphi, \varphi\right\rangle$ is positive definite on $C_{0}^{\infty}\left(\mathbb{R}^{+}\right)$. It is natural to take the Friedrichs extension of $H_{c}$, thereby defining a self-adjoint operator in $L^{2}\left(\mathbb{R}^{+}\right)$, which generates an isometry group in $H_{0}^{1}\left(\mathbb{R}^{+}\right)$.

Local well-posedness for parameters $1<p<\infty$ and $0<c<\frac{1}{4}$ follows by standard arguments (see e.g. in [3] Chapter 4). In particular, the following holds.

Theorem 1.1. Let $1<p<\infty$ and $c<1 / 4$. For any initial value $u_{0} \in$ $H_{0}^{1}\left(\mathbb{R}^{+}\right)$, there exist $T_{\min }, T_{\max } \in(0, \infty]$ and a unique maximal solution $u \in$ $C\left(\left(-T_{\min }, T_{\max }\right), H_{0}^{1}\left(\mathbb{R}^{+}\right)\right)$of $(1.1)$, which satisfies for all $t \in\left(-T_{\min }, T_{\max }\right)$ the conservation laws

$$
\|u(t)\|_{L^{2}}=\left\|u_{0}\right\|_{L^{2}}, \quad E(u(t))=E\left(u_{0}\right),
$$

where the energy is defined as

$$
E(u)=\frac{1}{2}\left\|u^{\prime}\right\|_{L^{2}}^{2}-\frac{c}{2}\left\|\frac{u}{x}\right\|_{L^{2}}^{2}-\frac{1}{p+1}\|u\|_{L^{p+1}}^{p+1}, \text { for } u \in H_{0}^{1}\left(\mathbb{R}^{+}\right) .
$$

Moreover, the so-called blow-up alternative holds: if $T_{\max }<\infty$ then $\lim _{t \rightarrow T_{\max }}\left\|u^{\prime}(t)\right\|_{L^{2}}=\infty,\left(\right.$ or $T_{\min }<\infty$ then $\left.\lim _{t \rightarrow-T_{\min }}\left\|u^{\prime}(t)\right\|_{L^{2}}=\infty\right)$.

In this work we address the existence of standing wave solutions and their orbital stability/instability. By introducing the ansatz $u(t, x)=e^{i \omega t} \varphi(x)$, the standing wave equation to (1.1) reads as

$$
\varphi^{\prime \prime}+\frac{c}{x^{2}} \varphi-\omega \varphi+|\varphi|^{p-1} \varphi=0 .
$$


First we will prove regularity of standing waves and the Pohozaev identities. To establish the existence of standing waves we carry out a minimization procedure on the Nehari manifold for the so-called action functional

$$
S(v)=\frac{1}{2}\left\|v^{\prime}\right\|_{L^{2}}^{2}-\frac{c}{2}\left\|\frac{v}{x}\right\|_{L^{2}}^{2}+\frac{\omega}{2}\|v\|_{L^{2}}^{2}-\frac{1}{p+1}\|v\|_{L^{p+1}}^{p+1} \quad v \in H_{0}^{1}\left(\mathbb{R}^{+}\right) .
$$

Owing to the non-compactness of the problem, we have to use a profile decomposition lemma, in the spirit of the article by Jeanjean and Tanaka [11]. To establish strong convergence of the minimizing sequence on the Nehari manifold we compare the minimization problem with the problem "at infinity", i.e. when $c=0$. Hence, we obtain that the set of bound states is not empty:

$$
\mathcal{A}=\left\{u \in H_{0}^{1}\left(\mathbb{R}^{+}\right) \backslash\{0\}: u^{\prime \prime}+c u / x^{2}-\omega u+|u|^{p-1} u=0\right\} \neq \varnothing .
$$

We are in particular interested in the orbital stability/instability of ground states, i.e., solutions which minimize the action functional. We denote the set of ground sate solutions by

$$
\mathcal{G}=\{u \in \mathcal{A}: S(u) \leqslant S(v) \text { for all } v \in \mathcal{A}\} .
$$

We use Lions' concentration-compactness principle to obtain a variational characterization of ground states on an $L^{2}$-constraint, thereby establishing the orbital stability of the set of ground states for nonlinearities with power $1<p<5$. Finally, for $p \geqslant 5$ we establish strong instability by a convexity argument.

\section{Existence of bound states}

We start by investigating the standing wave equation,

$$
\left\{\begin{array}{l}
\varphi^{\prime \prime}+\frac{c}{x^{2}} \varphi-\omega \varphi+|\varphi|^{p-1} \varphi=0, \\
\varphi \in H_{0}^{1}\left(\mathbb{R}^{+}\right) \backslash\{0\} .
\end{array}\right.
$$

First, we prove the regularity of solutions to (2.1) by a bootstrap argument.

Proposition 2.1. Let $\omega>0$ and $c<1 / 4$. Assume $\varphi \in H_{0}^{1}\left(\mathbb{R}^{+}\right)$is a solution of (2.1) in $H^{-1}\left(\mathbb{R}^{+}\right)$. Then the following statements are true

(1) $\varphi \in W_{0}^{2, r}((\epsilon, \infty))$ for all $r \in[2,+\infty)$ and $\epsilon>0$, in particular $\varphi \in$ $C^{1}((\epsilon, \infty))$;

(2) The solution is exponentially bounded, that is $\mathrm{e}^{\sqrt{\omega} x}\left(|\varphi|+\left|\varphi^{\prime}\right|\right) \in L^{\infty}\left(\mathbb{R}^{+}\right)$;

Proof. (1) For $\varphi \in H_{0}^{1}\left(\mathbb{R}^{+}\right)$we have $\varphi \in L^{q}\left(\mathbb{R}^{+}\right)$for all $q \in[2, \infty]$. We get easily that $|\varphi|^{p-1} \varphi \in L^{q}\left(\mathbb{R}^{+}\right)$for all $q \in[2, \infty)$. By (2.1) we have for any $\epsilon>0$ that $\varphi \in W_{0}^{2, q}((\epsilon, \infty))$ for all $q \in[2, \infty)$. By Sobolev's embedding we get $\varphi \in C^{1, \delta}((\epsilon, \infty))$ for all $\delta \in(0,1)$, hence $|\varphi(x)| \rightarrow 0$, and $\left|\varphi^{\prime}(x)\right| \rightarrow 0$ as $x \rightarrow \infty$.

(2) Let $\omega>0$. Changing $\varphi(x)$ to $\varphi(x)=\omega^{1 /(p-1)} \varphi(\sqrt{\omega} x)$ we may assume that $\omega=1$ in $(2.1)$. Let $\varepsilon>0$ and $\theta_{\varepsilon}(x)=e^{\frac{x}{1+\varepsilon x}}$, for $x \geqslant 0$. It is easy to see that $\theta_{\varepsilon}$ is bounded, Lipschitz continuous, and $\left|\theta_{\varepsilon}^{\prime}(x)\right| \leqslant \theta_{\varepsilon}(x)$ for all $x \in \mathbb{R}^{+}$. 
Additionally, $\theta_{\varepsilon}(x) \rightarrow e^{x}$ uniformly on bounded sets of $\mathbb{R}^{+}$. Taking the scalar product of the equation (2.1) with $\theta_{\varepsilon} \varphi \in H_{0}^{1}\left(\mathbb{R}^{+}\right)$, we get

$$
\operatorname{Re} \int_{\mathbb{R}^{+}} \varphi^{\prime} \cdot\left(\theta_{\varepsilon} \bar{\varphi}\right)^{\prime} d x-c \int_{\mathbb{R}^{+}} \theta_{\varepsilon} \frac{|\varphi|^{2}}{x^{2}} d x+\int_{\mathbb{R}^{+}} \theta_{\varepsilon}|\varphi|^{2} d x=\int_{\mathbb{R}^{+}} \theta_{\varepsilon}|\varphi|^{p+1} d x .
$$

Using the inequality $\operatorname{Re}\left(\varphi^{\prime}\left(\theta_{\varepsilon} \bar{\varphi}\right)^{\prime}\right) \geqslant \theta_{\varepsilon}\left|\varphi^{\prime}\right|^{2}-\theta_{\varepsilon}|\varphi|\left|\varphi^{\prime}\right|$ and

$$
\int_{\mathbb{R}^{+}} \theta_{\varepsilon}|\varphi|\left|\varphi^{\prime}\right| d x \leqslant \frac{1}{2} \int_{\mathbb{R}^{+}} \theta_{\varepsilon}|\varphi|^{2} d x+\frac{1}{2} \int_{\mathbb{R}^{+}} \theta_{\varepsilon}\left|\varphi^{\prime}\right|^{2} d x,
$$

we obtain

$$
\frac{1}{2} \int_{\mathbb{R}^{+}} \theta_{\varepsilon}\left|\varphi^{\prime}\right|^{2} d x+\frac{1}{2} \int_{\mathbb{R}^{+}} \theta_{\varepsilon}|\varphi|^{2} d x-c \int_{\mathbb{R}^{+}} \theta_{\varepsilon} \frac{|\varphi|^{2}}{x^{2}} d x \leqslant \int_{\mathbb{R}^{+}} \theta_{\varepsilon}|\varphi|^{p+1} d x .
$$

Let $R>0$ such that if $x>R$, then $\frac{c}{x^{2}} \leqslant \frac{1}{8}$ and $|\varphi(x)|^{p-1} \leqslant \frac{1}{8}$. Then we get

$$
\begin{aligned}
& c \int_{\mathbb{R}^{+}} \theta_{\varepsilon} \frac{|\varphi|^{2}}{x^{2}} d x+\int_{\mathbb{R}^{+}} \theta_{\varepsilon}|\varphi|^{p+1} \\
& \quad \leqslant e^{R}\left(\int_{0}^{R} c \frac{|\varphi|^{2}}{x^{2}} d x+\int_{0}^{R}|\varphi|^{p+1} d x\right)+\frac{1}{4} \int_{\mathbb{R}^{+}} \theta_{\varepsilon}|\varphi|^{2} d x .
\end{aligned}
$$

From the last two inequalities it follows that

$$
\frac{1}{2} \int_{\mathbb{R}^{+}} \theta_{\varepsilon}\left|\varphi^{\prime}\right|^{2} d x+\frac{1}{4} \int_{\mathbb{R}^{+}} \theta_{\varepsilon}|\varphi|^{2} d x \leqslant e^{R}\left(\int_{0}^{R} c \frac{|\varphi|^{2}}{x^{2}} d x+\int_{0}^{R}|\varphi|^{p+1} d x\right) .
$$

By taking $\varepsilon \downarrow 0$ we get

$$
\frac{1}{2} \int_{\mathbb{R}^{+}} e^{x}\left|\varphi^{\prime}\right|^{2} d x+\frac{1}{4} \int_{\mathbb{R}^{+}} e^{x}|\varphi|^{2} d x<\infty .
$$

Since both $\varphi$ and $\varphi^{\prime}$ are Lipschitz continuous we deduce that $|\varphi(x)| e^{x}$ and $\left|\varphi^{\prime}(x)\right| e^{x}$ are bounded.

We now prove that there exists a solution to (2.1). We define the action functional associated to (2.1) as follows

$$
S(u)=\frac{1}{2} H(u)+\frac{\omega}{2}\|u\|_{L^{2}}^{2}-\frac{1}{p+1}\|u\|_{L^{p+1}}^{p+1},
$$

for $c<1 / 4$ and $u \in H_{0}^{1}\left(\mathbb{R}^{+}\right)$. Clearly, we have

$$
S^{\prime}(u)=-u^{\prime \prime}-\frac{c}{x^{2}} u+\omega u-|u|^{p-1} u \text {. }
$$

Therefore, to prove the existence of a solution to (2.1) amounts to show that $S$ has a nontrivial critical point. A simple calculation yields the following identities.

Lemma 2.2. Assume $p>1, \omega>0$ and $c<1 / 4$. Let $\varphi \in H_{0}^{1}\left(\mathbb{R}^{+}\right)$be a solution of (2.1) in $H^{-1}\left(\mathbb{R}^{+}\right)$. Then the following identities are true:

$$
\begin{gathered}
\left\|\varphi^{\prime}\right\|_{L^{2}}^{2}-c\left\|\frac{\varphi}{x}\right\|_{L^{2}}^{2}+\omega\|\varphi\|_{L^{2}}^{2}-\|\varphi\|_{L^{p+1}}^{p+1}=0 \\
\left\|\varphi^{\prime}\right\|_{L^{2}}^{2}-c\left\|\frac{\varphi}{x}\right\|_{L^{2}}^{2}-\frac{p-1}{2(p+1)}\|\varphi\|_{L^{p+1}}^{p+1}=0 .
\end{gathered}
$$


Proof. We obtain the first equality by multiplying (2.1) by $\bar{\varphi}$ and integrating over $\mathbb{R}^{+}$.

To prove the second equality, let us put $\varphi_{\lambda}(x)=\lambda^{1 / 2} \varphi(\lambda x)$ for $\lambda>0$. We have that

$$
S\left(\varphi_{\lambda}\right)=\frac{\lambda^{2}}{2}\left\|\varphi^{\prime}\right\|_{L^{2}}^{2}-\frac{\lambda^{2} c}{2}\left\|\frac{\varphi}{x}\right\|_{L^{2}}^{2}+\frac{\omega}{2}\|\varphi\|_{L^{2}}^{2}-\frac{\lambda^{(p-1) / 2}}{p+1}\|\varphi\|_{L^{p+1}}^{p+1},
$$

from which we get

$$
\left.\frac{\partial}{\partial \lambda} S\left(\varphi_{\lambda}\right)\right|_{\lambda=1}=\left\|\varphi^{\prime}\right\|_{L^{2}}^{2}-c\left\|\frac{\varphi}{x}\right\|_{L^{2}}^{2}-\frac{p-1}{2(p+1)}\|\varphi\|_{L^{p+1}}^{p+1}
$$

We also have that

$$
\left.\frac{\partial}{\partial \lambda} S\left(\varphi_{\lambda}\right)\right|_{\lambda=1}=\left\langle S^{\prime}(\varphi),\left.\frac{\partial \varphi_{\lambda}}{\partial \lambda}\right|_{\lambda=1}\right\rangle
$$

Now $\left.\frac{\partial \varphi_{\lambda}}{\partial \lambda}\right|_{\lambda=1}=\frac{1}{2} \varphi+x \varphi^{\prime}$ is in $H^{1}\left(\mathbb{R}^{+}\right)$, since $\varphi$ and $\varphi^{\prime}$ are exponentially decaying at infinity by Proposition 2.1. We obtain that the right hand-side is well-defined. Since $\varphi$ is a critical point of $S$, we obtain $S^{\prime}(\varphi)=0$, which concludes the proof.

Remark 2.3. Since (2.2) and (2.3) hold for solutions of (2.1), it follows for $\omega \neq 0$ that

$$
\omega\|\varphi\|_{L^{2}}^{2}=\frac{p+3}{2(p+1)}\|\varphi\|_{L^{p+1}}^{p+1}>0 .
$$

Hence, non-trivial solution of (2.1) exists only if $\omega>0$.

Let us define for all $u \in H_{0}^{1}\left(\mathbb{R}^{+}\right)$the following functional:

$$
J(u)=\left(S^{\prime}(u), u\right)_{H^{-1}, H_{0}^{1}}=H(u)+\omega\|u\|_{L^{2}}^{2}-\|u\|_{L^{p+1}}^{p+1} .
$$

It follows from Lemma 2.2 , that $\mathcal{N}=\left\{u \in H_{0}^{1}\left(\mathbb{R}^{+}\right) \backslash\{0\}: J(u)=0\right\}$ contains all nontrivial critical points of $S$. We aim to show that the infimum of the following minimization problem is attained

$$
m=\inf \{S(u): u \in \mathcal{N}\}=\frac{p-1}{2(p+1)} \inf \left\{\|u\|_{L^{p+1}}^{p+1}: u \in \mathcal{N}\right\} .
$$

First we prove the following lemma.

Lemma 2.4. $\mathcal{N}$ is nonempty, and $m>0$.

Proof. Let $u \in H_{0}^{1}\left(\mathbb{R}^{+}\right) \backslash\{0\}$. Take

$$
t(u)=\left(\frac{H(u)+\omega\|u\|_{L^{2}}^{2}}{\|u\|_{L^{p+1}}^{p+1}}\right)^{1 /(p-1)} .
$$

By simple calculation, we get that $J(t(u) u)=0$, hence $t(u) u \in \mathcal{N}$. We see that

$$
m=\inf _{u \in \mathcal{N}} S(u)=\inf _{u \in \mathcal{N}}\left(S(u)-\frac{1}{p+1} J(u)\right)=\frac{p-1}{2(p+1)} \inf _{u \in \mathcal{N}^{\prime}}\left(H(u)+\omega\|u\|_{L^{2}}^{2}\right) .
$$


It follows from Sobolev's and Hardy's inequalities, that there exists $C>0$ such that

$$
H(u)+\omega\|u\|_{L^{2}}^{2}=\|u\|_{L^{p+1}}^{p+1} \leqslant C\left(H(u)+\omega\|u\|_{L^{2}}^{2}\right)^{(p+1) / 2},
$$

for all $u \in \mathcal{N}$. Hence,

$$
\left(\frac{1}{C}\right)^{2 /(p-1)} \leqslant H(u)+\omega\|u\|_{L^{2}}^{2} \text { for all } u \in H_{0}^{1}\left(\mathbb{R}^{+}\right),
$$

which implies that

$$
m \geqslant \frac{p-1}{2(p+1)}\left(\frac{1}{C}\right)^{2 /(p-1)}>0
$$

Lemma 2.5. Let $c<1 / 4$, and $p>1$. Then if $u \in H_{0}^{1}\left(\mathbb{R}^{+}\right)$is a minimizer of (2.4), then $|u|$ is also a minimizer. In particular, we can search for the minimizers of (2.4) among the non-negative, real-valued functions of $H_{0}^{1}\left(\mathbb{R}^{+}\right)$.

Proof. Let $u \in H_{0}^{1}\left(\mathbb{R}^{+}\right)$be a solution of the minimization problem (2.4). It is well-known that if $u \in H_{0}^{1}\left(\mathbb{R}^{+}\right)$then $|u| \in H_{0}^{1}\left(\mathbb{R}^{+}\right)$and $\left\||u|^{\prime}\right\|_{L^{2}} \leqslant\left\|u^{\prime}\right\|_{L^{2}}$. Moreover, $\||u|\|_{L^{p+1}}=\|u\|_{L^{p+1}}$. Therefore, $J(|u|) \leqslant J(u)$. Hence there exists a $\lambda \in(0,1]$ such that $J(\lambda|u|)=J(u)=0$. Then

$$
m \leqslant S(\lambda|u|)=\frac{p-1}{2(p+1)}\|\lambda u\|_{L^{p+1}}^{p+1} \leqslant \frac{p-1}{2(p+1)}\|u\|_{L^{p+1}}^{p+1}=m .
$$

Hence $\lambda=1, J(|u|)=0$, and $S(|u|)=m$.

Let $m \in \mathbb{R}$. We say that $\left\{u_{n}\right\}_{n \in \mathbb{N}}$ is a Palais-Smale sequence for $S$ at level $m$, if

$$
S\left(u_{n}\right) \rightarrow m, \quad S^{\prime}\left(u_{n}\right) \rightarrow 0 \text { in } H^{-1}\left(\mathbb{R}^{+}\right),
$$

as $n \rightarrow \infty$.

Lemma 2.6. Let $c<1 / 4$, and $p>1$. There exists a bounded Palais-Smale sequence $\left\{u_{n}\right\}_{n \in \mathbb{N}} \subset \mathcal{N}$ for $S$ at the level $m$. Namely, there is a sequence $\left\{u_{n}\right\}_{n \in \mathbb{N}} \subset \mathcal{N}$ bounded in $H^{1}\left(\mathbb{R}^{+}\right)$such that, as $n \rightarrow \infty$,

$$
S\left(u_{n}\right) \rightarrow m, \quad S^{\prime}\left(u_{n}\right) \rightarrow 0 \text { in } H^{-1}\left(\mathbb{R}^{+}\right) .
$$

Proof. Since $\mathcal{N}$ is a closed manifold in $H_{0}^{1}\left(\mathbb{R}^{+}\right)$, it is a complete metric space. Hence, Ekeland's variational principle (see pp. 51-53 in [20]) directly yields the existence of a Palais-Smale sequence at level $m$ in $\mathcal{N}$.

We now show that if $\left\{u_{n}\right\}_{n \in \mathbb{N}} \subset \mathcal{N}$ and $\left\|u_{n}\right\|_{H^{1}}^{2} \rightarrow \infty$, then $S\left(u_{n}\right) \rightarrow \infty$. Indeed, since $u_{n} \in \mathcal{N}$ from Hardy's inequality we get that

$$
\begin{aligned}
S\left(u_{n}\right) & =\frac{p-1}{2(p+1)}\left(H\left(u_{n}\right)+\omega\left\|u_{n}\right\|_{L^{2}}^{2}\right) \\
& \geqslant \frac{p-1}{2(p+1)}\left(\min \{1,(1-4 c)\}\left\|u_{n}^{\prime}\right\|_{L^{2}}^{2}+\omega\left\|u_{n}\right\|_{L^{2}}^{2}\right) .
\end{aligned}
$$

Therefore, any Palais-Smale sequence $\left\{u_{n}\right\}_{n \in \mathbb{N}}$ is bounded in $H_{0}^{1}\left(\mathbb{R}^{+}\right)$. 
Before proceeding to our next lemma, let us recall some classical results, see e.g. [3], concerning the case $c=0$. It is well-known that the set of solutions of

$$
q^{\prime \prime}-\omega q+|q|^{p-1} q=0, \quad \omega>0, \quad q \in H^{1}(\mathbb{R})
$$

is given by $\left\{e^{i \theta} q(\cdot+y): y \in \mathbb{R}, \theta \in \mathbb{R}\right\}$, where $q$ is a symmetric, positive solution of (2.5), explicitly given by

$$
q(x)=\left(\frac{(p+1) \omega}{2} \operatorname{sech}^{2}\left(\frac{(p-1) \sqrt{\omega}}{2} x\right)\right)^{1 /(p-1)}
$$

Moreover, up to translation and phase invariance, it is the unique solution of the minimization problem

$$
\begin{aligned}
m^{\infty} & =\inf \left\{S^{\infty}(u): u \in H^{1}(\mathbb{R}) \backslash\{0\}, J^{\infty}(u)=0\right\} \\
& =\frac{p-1}{2(p+1)} \inf \left\{\|u\|_{L^{p+1}(\mathbb{R})}^{p+1}: u \in H^{1}(\mathbb{R}) \backslash\{0\}, J^{\infty}(u)=0\right\},
\end{aligned}
$$

where the functionals $S^{\infty}$ and $J^{\infty}$ are defined by

$$
\begin{aligned}
& S^{\infty}(u)=\frac{1}{2}\left\|u^{\prime}\right\|_{L^{2}(\mathbb{R})}^{2}+\frac{\omega}{2}\|u\|_{L^{2}(\mathbb{R})}^{2}-\frac{1}{p+1}\|u\|_{L^{p+1}(\mathbb{R})}^{p+1}, \\
& J^{\infty}(u)=\left\|u^{\prime}\right\|_{L^{2}(\mathbb{R})}^{2}+\omega\|u\|_{L^{2}(\mathbb{R})}^{2}-\|u\|_{L^{p+1}(\mathbb{R})}^{p+1} .
\end{aligned}
$$

Lemma 2.7. Let $0<c<1 / 4$, and $p>1$. Then $m<m^{\infty}$.

Proof. It is not hard to see that $m \leqslant m^{\infty}$, we only need to prove that $m \neq m^{\infty}$. Let us first note that if $u \in H_{0}^{1}\left(\mathbb{R}^{+}\right) \backslash\{0\}$ and $J(u)<0$, then $m<\tilde{S}(u)$, where

$$
\tilde{S}(u)=\frac{p-1}{2(p+1)}\left(H(u)+\omega\|u\|_{L^{2}}^{2}\right) .
$$

Indeed, if $J(u)<0$, then let us define

$$
t(u)=\left(\frac{H(u)+\omega\|u\|_{L^{2}}^{2}}{\|u\|_{L^{p+1}}^{p+1}}\right)^{1 /(p-1)} .
$$

Hence $t(u) \in(0,1), t(u) u \in \mathcal{N}$, and

$$
m \leqslant \tilde{S}(t(u) u)=t^{2}(u) \tilde{S}(u)<\tilde{S}(u) .
$$

Now let us define $\psi_{A}(x)=q(x+A)-q(x-A)$ for $x \geqslant 0$. For large enough $A$ we obtain the following estimates (see Lemma 5.1 in the Appendix):

$$
\begin{aligned}
\int_{0}^{\infty}\left|\psi_{A}^{\prime}\right|^{2} d x & =\int_{-\infty}^{\infty}\left|q^{\prime}\right|^{2} d x+O\left(\left(2 A+\frac{1}{\sqrt{\omega}}\right) e^{-2 \sqrt{\omega} A}\right), \\
\int_{0}^{\infty}\left|\psi_{A}\right|^{2} d x & =\int_{-\infty}^{\infty}|q|^{2} d x+O\left(\left(2 A+\frac{1}{\sqrt{\omega}}\right) e^{-2 \sqrt{\omega} A}\right), \\
\int_{0}^{\infty} \frac{\left|\psi_{A}\right|^{2}}{x^{2}} d x & \leqslant \frac{4}{A^{2}} \int_{-\infty}^{\infty}|q|^{2} d x+O\left(\frac{1}{A^{2}} e^{-\sqrt{\omega} A}\right), \\
\int_{0}^{\infty}\left|\psi_{A}\right|^{p+1} d x & =\int_{-\infty}^{\infty}|q|^{p+1} d x+O\left(e^{-2 \sqrt{\omega} A}\right) .
\end{aligned}
$$


Since $0<c<1 / 4$, we obtain for $A>0$ large enough

$$
\begin{aligned}
J\left(\psi_{A}\right) & \leqslant\left\|q^{\prime}\right\|_{L^{2}(\mathbb{R})}^{2}+\omega\|q\|_{L^{2}(\mathbb{R})}^{2}-\|q\|_{L^{p+1}(\mathbb{R})}^{p+1}-\frac{4 c}{A^{2}}\|q\|_{L^{2}(\mathbb{R})}^{2}+O\left(\frac{1}{A^{2}} e^{-\sqrt{\omega} A}\right) \\
& =-\frac{4 c}{A^{2}}\|q\|_{L^{2}(\mathbb{R})}^{2}+O\left(\frac{1}{A^{2}} e^{-\sqrt{\omega} A}\right)<0,
\end{aligned}
$$

and

$$
\begin{aligned}
\tilde{S}\left(\psi_{A}\right) & \leqslant \frac{p-1}{2(p+1)}\left(\left\|q^{\prime}\right\|_{L^{2}(\mathbb{R})}^{2}+\omega\|q\|_{L^{2}(\mathbb{R})}^{2}-\frac{4 c}{A^{2}}\|q\|_{L^{2}(\mathbb{R})}^{2}\right)+O\left(\frac{1}{A^{2}} e^{-\sqrt{\omega} A}\right) \\
& =m^{\infty}-\frac{p-1}{2(p+1)} \frac{4 c}{A^{2}}\|q\|_{L^{2}(\mathbb{R})}^{2}+O\left(\frac{1}{A^{2}} e^{-\sqrt{\omega} A}\right)<m^{\infty} .
\end{aligned}
$$

Since $J\left(\psi_{A}\right)<0$, we get

$$
m<\tilde{S}\left(\psi_{A}\right)<m^{\infty}
$$

which concludes the proof.

We need the following lemma, which describes the behavior of bounded Palais-Smale sequences. We note that $H_{0}^{1}\left(\mathbb{R}^{+}\right)$functions can be extended to functions in $H^{1}(\mathbb{R})$ by setting $u \equiv 0$ on $\mathbb{R}^{-}$. The proof of the following statement is presented in the appendix.

Lemma 2.8. Let $\left\{u_{n}\right\}_{n \in \mathbb{N}} \subset H_{0}^{1}\left(\mathbb{R}^{+}\right)$be a bounded Palais-Smale sequence for $S$ at level $m$. Then there exists a subsequence still denoted by $\left\{u_{n}\right\}_{n \in \mathbb{N}}, a$ $u_{0} \in H_{0}^{1}\left(\mathbb{R}^{+}\right)$solution of

$$
\varphi^{\prime \prime}+\frac{c}{x^{2}} \varphi-\omega \varphi+|\varphi|^{p-1} \varphi=0,
$$

an integer $k \geqslant 0,\left\{x_{n}^{i}\right\}_{i=1}^{k} \subset \mathbb{R}^{+}$, and nontrivial solutions $q_{i}$ of (2.5) satisfying

$$
\begin{aligned}
& u_{n} \rightarrow u_{0} \quad \text { weakly in } H_{0}^{1}\left(\mathbb{R}^{+}\right), \\
& S\left(u_{n}\right) \rightarrow S\left(u_{0}\right)+\sum_{i=1}^{k} S^{\infty}\left(q_{i}\right), \\
& u_{n}-\left(u_{0}+\sum_{i=1}^{k} q_{i}\left(x-x_{n}^{i}\right)\right) \rightarrow 0 \quad \text { strongly in } H^{1}(\mathbb{R}), \\
& \left|x_{n}^{i}\right| \rightarrow \infty, \quad\left|x_{n}^{i}-x_{n}^{j}\right| \rightarrow \infty \quad \text { for } \quad 1 \leqslant i \neq j \leqslant k,
\end{aligned}
$$

where in case $k=0$, the above holds without $q_{i}$ and $x_{n}^{i}$.

We only need to show that the critical point of $S$ provided by Lemma 2.8 is non-trivial.

Theorem 2.9. Let $0<c<1 / 4$. Then there exists $u \in \mathcal{N} \backslash\{0\}, u \geqslant 0$ a.e., such that $S(u)=m$. 
Proof. We only have to prove that the $\left\{u_{n}\right\}_{n \in \mathbb{N}}$ bounded Palais-Smale sequence obtained in Lemma 2.6 admits a strongly convergent subsequence. Assume that it is not the case. Using Lemma 2.8 we see that $k \geqslant 1$ and $u_{n}$ is weakly convergent to $u_{0}$ in $H_{0}^{1}\left(\mathbb{R}^{+}\right)$up to a subsequence. Then

$$
m=\lim _{n \rightarrow \infty} S\left(u_{n}\right) \geqslant S\left(u_{0}\right)+S^{\infty}(q)=S\left(u_{0}\right)+m^{\infty} .
$$

Now, $S\left(u_{0}\right) \geqslant 0$ since $J\left(u_{0}\right)=0$. Thus $m \geqslant m^{\infty}$, which contradicts Lemma 2.7. Hence $k=0$ and $u_{n} \rightarrow u_{0}$ in $H_{0}^{1}\left(\mathbb{R}^{+}\right)$.

Lemma 2.10. Let $p>1$ and $\omega>0$. There exists $a \mu>0$ such that

$$
\int_{0}^{\infty}|u|^{2} d x=\mu, \text { for every } u \in \mathcal{G} .
$$

The mass of ground state solutions is $\mu=\frac{m}{\omega} \frac{p+3}{p-1}$. Moreover, we have

$$
\|u\|_{L^{p+1}}^{p+1}=\frac{2(p+1)}{p-1} m, \text { and } H(u)=m \text { for every } u \in \mathcal{G} .
$$

Proof. Since $u \in \mathcal{G}$ is a solution of (2.1), it satisfies (2.2) and (2.3). By subtracting the two identities we get

$$
\omega\|u\|_{L^{2}}^{2}=\frac{p+3}{2(p+1)}\|u\|_{L^{p+1}}^{p+1} .
$$

Additionally, since $u$ is a ground state solution, it also solves the minimization problem (2.4). From (2.4) and (2.3) we get

$$
\omega\|u\|_{L^{2}}^{2}+\frac{p-5}{2(p+1)}\|u\|_{L^{p+1}}^{p+1}=2 m .
$$

From (2.7) and (2.8) it follows

$$
\|u\|_{L^{2}}^{2}=\frac{m}{\omega} \frac{p+3}{p-1}>0 .
$$

Thus, let $\mu=\frac{m}{\omega} \frac{p+3}{p-1}$. Now it follows from (2.4) and (2.3) that

$$
\|u\|_{L^{p+1}}^{p+1}=\frac{2(p+1)}{p-1} m, \text { and } H(u)=m \text { for every } u \in \mathcal{G} .
$$

which concludes the proof.

\section{Stability}

In this section we consider nonlinearities with $1<p<5$. Our aim is to prove orbital stability of the standing waves. To do so, we investigate the minimization problem:

$$
I=\inf \{E(u): u \in \Gamma\}
$$

where

$$
\Gamma=\left\{u \in H_{0}^{1}\left(\mathbb{R}^{+}\right):\|u\|_{L^{2}}^{2}=\mu\right\} .
$$

and the energy $E$ is defined by (1.4). We will rely on a of Lions' concentrationcompactness principle [15] and the arguments by Cazenave and Lions [4], see 
also in [3]. The main problem is to obtain compactness of minimizing sequences owing to the absence of translation invariance. We define the problem at infinity by

$$
I^{\infty}=\inf \left\{E^{\infty}(u): u \in H^{1}(\mathbb{R}) \text { and }\|u\|_{L^{2}}^{2}=\mu\right\}
$$

where

$$
E^{\infty}(u)=\frac{1}{2} \int_{\mathbb{R}}\left|u^{\prime}\right|^{2} d x-\frac{1}{p+1} \int_{\mathbb{R}}|u|^{p+1} d x .
$$

We recall some well-known facts about the minimization problem (3.2) (see [3, Chapter 8.]). For every $\mu>0$, there exists a unique, positive, symmetric function $q=q(\mu) \in H^{1}(\mathbb{R})$, such that

$$
\|q\|_{L^{2}}=\mu, \quad E^{\infty}(q)=I^{\infty},
$$

and $q$ solves the nonlinear equation

$$
q^{\prime \prime}-\lambda q+|q|^{p-1} q=0
$$

where $\lambda=\lambda(\mu)$. Moreover, there exists $M>0$ such that

$$
e^{\sqrt{\lambda}|x|}|q(x)| \leqslant M \text { and } e^{\sqrt{\lambda}|x|}\left|q^{\prime}(x)\right| \leqslant M .
$$

We proceed by proving the following lemma:

Lemma 3.1. If $0<c<1 / 4$, then the following inequality holds:

$$
I<I^{\infty}
$$

Proof. For $A>0$, let $C(A)$ be a normalizing factor specified later. Let us define

$$
\Psi_{A}(x)=C(A)(q(x+A)-q(x-A)) \text { for } x \geqslant 0 .
$$

Since $q$ is even, we obtain $\Psi_{A} \in H_{0}^{1}\left(\mathbb{R}^{+}\right)$and

$$
\int_{0}^{\infty}\left|\Psi_{A}(x)\right|^{2} d x=C^{2}(A)\left(\int_{-\infty}^{\infty}|q|^{2} d x-\int_{-\infty}^{\infty} q(x+A) q(x-A) d x\right) .
$$

We estimate the second integral by (see Lemma 5.1)

$$
\int_{-\infty}^{\infty} q(x+A) q(x-A) d x=O\left(\left(2 A+\frac{1}{\sqrt{\lambda}}\right) e^{-2 \sqrt{\lambda} A}\right) .
$$

We define

$$
C(A)=\left(\frac{\mu}{\mu-\int_{-\infty}^{\infty} q(x+A) q(x-A) d x}\right)^{1 / 2}
$$

$C(A)$ is a continuous function of $A, C(A) \geqslant 1$, and $C(A) \rightarrow 1$ exponentially fast as $A \rightarrow \infty$. Thus, $\left\|\Psi_{A}\right\|_{L^{2}}=\mu$ for all $A>0$. By Lemma 5.1 in the 
Appendix, we obtain for $A>0$ large enough that

$$
\begin{gathered}
\int_{0}^{\infty}\left|\Psi_{A}^{\prime}\right|^{2} d x=C^{2}(A) \int_{-\infty}^{\infty}\left|q^{\prime}\right| d x+O\left(\left(2 A+\frac{1}{\sqrt{\lambda}}\right) e^{-2 \sqrt{\lambda} A}\right) \\
\int_{0}^{\infty} \frac{\left|\Psi_{A}\right|^{2}}{x^{2}} d x \leqslant \frac{4 C^{2}(A)}{A^{2}} \int_{0}^{\infty}\left|\Psi_{A}\right|^{2} d x+O\left(\frac{1}{A^{2}} e^{-\sqrt{\lambda} A}\right) \\
\int_{0}^{\infty}\left|\Psi_{A}\right|^{p+1} d x=C^{p+1}(A) \int_{-\infty}^{\infty}|q|^{p+1} d x+O\left(e^{-2 \sqrt{\lambda} A}\right)
\end{gathered}
$$

Hence for $A$ large enough we get

$$
\begin{aligned}
E\left(\Psi_{A}\right)= & \frac{1}{2} \int_{0}^{\infty}\left|\Psi_{A}^{\prime}\right|^{2} d x-\frac{c}{2} \int_{0}^{\infty} \frac{\left|\Psi_{A}\right|^{2}}{x^{2}} d x-\frac{1}{p+1} \int_{0}^{\infty}\left|\Psi_{A}\right|^{p+1} d x \\
\leqslant & C^{2}(A)\left(\frac{1}{2} \int_{-\infty}^{\infty}\left|q^{\prime}\right|^{2} d x-\frac{C^{p-1}(A)}{p+1} \int_{-\infty}^{\infty}|q|^{p+1} d x\right) \\
& -\frac{c}{2} \frac{4 C^{2}(A)}{A^{2}} \int_{0}^{\infty}\left|\Psi_{A}\right|^{2} d x+O\left(\frac{1}{A^{2}} e^{-\sqrt{\lambda} A}\right) .
\end{aligned}
$$

Owing to the exponential decay of the last term, for large $A$ we get

$$
E\left(\Psi_{A}\right) \leqslant E(q)-\frac{2 c}{A^{2}} \mu=I^{\infty}-\frac{2 c}{A^{2}} \mu .
$$

Since $0<c<1 / 4$ we get that $E\left(\Psi_{A}\right)<I^{\infty}$, which concludes the proof.

We need the following version of the concentration-compactness principle. The proof follows the same way as in the classical case (see [15]).

Lemma 3.2. Let $0<c<1 / 4$, and $\left\{u_{n}\right\}_{n \in \mathbb{N}} \subset H_{0}^{1}\left(\mathbb{R}^{+}\right)$be a sequence satisfying

$$
\lim _{n \rightarrow \infty}\left\|u_{n}\right\|_{L^{2}}^{2}=M \text { and } \lim _{n \rightarrow \infty} H\left(u_{n}\right)<\infty \text {. }
$$

Then there exists a subsequence $\left\{u_{n}\right\}_{n \in \mathbb{N}}$ such that it satisfies one of the following alternatives.

(Vanishing) $\lim _{n \rightarrow \infty}\left\|u_{n}\right\|_{L^{p}} \rightarrow 0$ for all $p \in(2, \infty)$.

(Dichotomy) There are sequences $\left\{v_{n}\right\}_{n \in \mathbb{N}},\left\{w_{n}\right\}_{n \in \mathbb{N}}$ in $H_{0}^{1}\left(\mathbb{R}^{+}\right)$and a constant $\alpha \in(0,1)$ such that:

(1) $\operatorname{dist}\left(\operatorname{supp}\left(v_{n}\right), \operatorname{supp}\left(w_{n}\right)\right) \rightarrow \infty$;

(2) $\left|v_{n}\right|+\left|w_{n}\right| \leqslant\left|u_{n}\right|$;

(3) $\sup _{n \in \mathbb{N}}\left(\left\|v_{n}\right\|_{H^{1}}+\left\|w_{n}\right\|_{H^{1}}\right)<\infty$;

(4) $\left\|v_{n}\right\|_{L^{2}}^{2} \rightarrow \alpha M$ and $\left\|w_{n}\right\|_{L^{2}}^{2} \rightarrow(1-\alpha) M$ as $n \rightarrow \infty$;

(5) $\left.\lim _{n \rightarrow \infty}\left|\int_{0}^{\infty}\right| u_{n}\right|^{q} d x-\int_{0}^{\infty}\left|v_{n}\right|^{q} d x-\int_{0}^{\infty}\left|w_{n}\right|{ }^{q} d x \mid=0$ for all $q \in[2, \infty)$;

(6) $\liminf _{n \rightarrow \infty}\left\{H\left(u_{n}\right)-H\left(v_{n}\right)-H\left(w_{n}\right)\right\} \geqslant 0$.

(Compactness) There exists a sequence $y_{n} \in \mathbb{R}^{+}$, such that for any $\varepsilon>0$ there is an $R>0$ with the property that

$$
\int_{\left(y_{n}-R, y_{n}+R\right) \cap \mathbb{R}^{+}}\left|u_{n}\right|^{2} \geqslant M-\varepsilon .
$$

for all $n \in \mathbb{N}$.

We are now in a position to prove the following lemma. 
Lemma 3.3. Let $1<p<5,0<c<1 / 4$, and $\omega>0$. Then the infimum in (3.1) is attained. Additionally, all minimizing sequences are relatively compact, that is if $\left\{u_{n}\right\}_{n \in \mathbb{N}}$ satisfies $\left\|u_{n}\right\|_{L^{2}}^{2} \rightarrow \mu$ and $E\left(u_{n}\right) \rightarrow I$ then there exists a subsequence $\left\{u_{n}\right\}_{n \in \mathbb{N}}$ which converges to a minimizer $u \in H_{0}^{1}\left(\mathbb{R}^{+}\right)$.

Proof. Step 1. We first show that $0>I>-\infty$. Let $u \in \Gamma$. For $\lambda>0$, we define $u_{\lambda}(x)=\lambda^{1 / 2} u(\lambda x) \in \Gamma$. Clearly,

$$
E\left(u_{\lambda}\right)=\frac{\lambda^{2}}{2}\left\|u^{\prime}\right\|_{L^{2}}^{2}-\frac{c \lambda^{2}}{2} \int_{0}^{\infty} \frac{|u|^{2}}{x^{2}} d x-\frac{\lambda^{(p-1) / 2}}{p+1}\|u\|_{L^{p+1}}^{p+1}
$$

Since $1<p<5$, we can choose a small $\lambda>0$ such that $E\left(u_{\lambda}\right)<0$. Hence $I<0$.

Since $c \in(0,1 / 4)$, we have $H(u) \sim\left\|u^{\prime}\right\|_{L^{2}}^{2}$. We get from the GagliardoNirenberg inequality that there exists $C>0$ such that for all $u \in H_{0}^{1}\left(\mathbb{R}^{+}\right)$

$$
\int_{0}^{\infty}|u|^{p+1} d x \leqslant C H(u)^{\frac{p-1}{4}}\left(\int_{0}^{\infty}|u|^{2} d x\right)^{1+\frac{p-1}{4}} .
$$

Since $1<p<5$, this yields that there exists $\delta>0$ and $K>0$ such that

$$
E(u) \geqslant \delta\|u\|_{H^{1}}^{2}-K \text { for all } u \in \Gamma
$$

from which follows that $I>-\infty$.

Every minimizing sequence is bounded in $H_{0}^{1}\left(\mathbb{R}^{+}\right)$and bounded from below in $L^{p+1}\left(\mathbb{R}^{+}\right)$. Indeed, let $\left\{u_{n}\right\}_{n \in \mathbb{N}} \subset \Gamma$ be a minimizing sequence, then by $(3.3)$ it is bounded in $H_{0}^{1}\left(\mathbb{R}^{+}\right)$. Furthermore, for $n$ large enough we have $E\left(u_{n}\right)<I / 2$, thus

$$
\left\|u_{n}\right\|_{L^{p+1}}^{p+1}>-\frac{p+1}{2} I .
$$

Now $I<0$, hence the result follows.

Step 2. We now verify that all minimizing sequences have a subsequence which converges to a limit $u$ in $H_{0}^{1}\left(\mathbb{R}^{+}\right)$. Let $\left\{u_{n}\right\}_{n \in \mathbb{N}}$ satisfy $\left\|u_{n}\right\|_{L^{2}}^{2} \rightarrow \mu$ and $E\left(u_{n}\right) \rightarrow I$. Since every minimizing sequence is bounded in $H_{0}^{1}\left(\mathbb{R}^{+}\right),\left\{u_{n}\right\}_{n \in \mathbb{N}}$ has a weak-limit $u \in L^{p}\left(\mathbb{R}^{+}\right)$. We can apply the concentration-compactness principle (see Lemma 3.2) to the sequence $\left\{u_{n}\right\}_{n \in \mathbb{N}}$. We note that since the sequence is bounded from below in $L^{p+1}\left(\mathbb{R}^{+}\right)$vanishing cannot occur.

Now let us assume that dichotomy occurs. Let $\alpha \in(0,1),\left\{v_{n}\right\}_{n \in \mathbb{N}}$ and $\left\{w_{n}\right\}_{n \in \mathbb{N}}$ sequences as in Lemma 3.2. It follows from (5) and (6) of Lemma 3.2 that

$$
\liminf _{n \rightarrow \infty}\left(E\left(u_{n}\right)-E\left(v_{n}\right)-E\left(w_{n}\right)\right) \geqslant 0
$$

hence

$$
\limsup _{n \rightarrow \infty}\left(E\left(v_{n}\right)+E\left(w_{n}\right)\right) \leqslant I .
$$

Observe that for $u \in H_{0}^{1}\left(\mathbb{R}^{+}\right)$, and $a>0$, we have

$$
E(u)=\frac{1}{a^{2}} E(a u)+\frac{a^{p-1}-1}{p+1} \int_{0}^{\infty}|u|^{p+1} d x .
$$


Let $a_{n}=\sqrt{\mu} /\left\|v_{n}\right\|_{L^{2}}$ and $b_{k}^{2}=\sqrt{\mu} /\left\|w_{n}\right\|_{L^{2}}$. Hence, $a_{n} v_{n} \in \Gamma$ and $b_{n} w_{n} \in \Gamma$, which implies

$$
\begin{aligned}
& E\left(v_{n}\right) \geqslant \frac{I}{a_{n}^{2}}+\frac{a_{n}^{p-1}-1}{p+1} \int_{0}^{\infty}\left|v_{n}\right|^{p+1} d x, \\
& E\left(w_{n}\right) \geqslant \frac{I}{b_{n}^{2}}+\frac{b_{n}^{p-1}-1}{p+1} \int_{0}^{\infty}\left|w_{n}\right|^{p+1} d x .
\end{aligned}
$$

Therefore

$$
E\left(v_{n}\right)+E\left(w_{n}\right) \geqslant I\left(a_{n}^{-2}+b_{n}^{-2}\right)+\frac{a_{n}^{p-1}}{p+1} \int_{0}^{\infty}\left|v_{n}\right|^{p+1}+\frac{b_{n}^{p-1}}{p+1} \int_{0}^{\infty}\left|w_{n}\right|^{p+1} .
$$

Now we observe $a_{n}^{-2} \rightarrow \alpha$ and $b_{n}^{-2} \rightarrow(1-\alpha)$ by (4) of Lemma 3.2. Since $\alpha \in(0,1)$, we get that $\left.\theta=\min \left\{\alpha^{-(p-1) / 2} ;(1-\alpha)^{-(p-1) / 2}\right)\right\}>1$. Property (5) of Lemma 3.2 and (3.4) implies

$$
\liminf _{n \rightarrow \infty}\left(E\left(v_{n}\right)+E\left(w_{n}\right)\right) \geqslant I+\frac{\theta-1}{p+1} \liminf _{n \rightarrow \infty} \int_{0}^{\infty}\left|u_{n}\right|^{p+1} d x, \geqslant I+\frac{\theta-1}{2}>I,
$$

which contradicts (3.5). Hence the following holds: there exists a sequence $y_{n} \in \mathbb{R}^{+}$, such that for any $\varepsilon>0$ there exists $R>0$ with the property that

$$
\int_{\left(y_{n}-R, y_{n}+R\right) \cap \mathbb{R}^{+}}\left|u_{n}\right|^{2} \geqslant \mu-\varepsilon .
$$

for all $n \in \mathbb{N}$.

We now show that $\left\{y_{n}\right\}_{n \in \mathbb{N}}$ is bounded in $\mathbb{R}^{+}$. First we show that if $y_{n} \rightarrow \infty$, then

$$
\lim _{n \rightarrow \infty} \int_{0}^{\infty} \frac{\left|u_{n}\right|^{2}}{x^{2}} d x=0 .
$$

Let us assume by contradiction that

$$
\int_{0}^{\infty} \frac{\left|u_{n}\right|^{2}}{x^{2}} d x \geqslant \delta>0
$$

which implies together with Hardy's inequality that

$$
H\left(u_{n}\right) \geqslant(1 / 4-c) \delta
$$

Let us take $\xi \in C^{\infty}\left(\mathbb{R}^{+}\right)$, such that for $\tilde{R}>0$ and $a>0$ we have that $\xi(r)=1$ for $0 \leqslant r \leqslant \tilde{R}, \xi(r)=0$ for $r \geqslant \tilde{R}+a$, and $\left\|\xi^{\prime}\right\|_{L^{\infty}} \leqslant 2 / a$. We introduce $u_{n, 1}=u_{n} \cdot \xi$ and $u_{n, 2}=u_{n} \cdot(1-\xi)$. Clearly, $u_{n, 1} \in H_{0}^{1}\left(\mathbb{R}^{+}\right), u_{n, 2} \in H_{0}^{1}\left(\mathbb{R}^{+}\right)$ and $u_{n}=u_{n, 1}+u_{n, 2}$. Moreover, the following inequalities hold

$$
\begin{aligned}
& \left|u_{n, 1}^{\prime}\right|^{2} \leqslant 2\left(4 a^{-2}\left|u_{n}\right|^{2}+\left|u_{n}^{\prime}\right|^{2}\right), \\
& \left|u_{n, 2}^{\prime}\right|^{2} \leqslant 2\left(4 a^{-2}\left|u_{n}\right|^{2}+\left|u_{n}^{\prime}\right|^{2}\right) .
\end{aligned}
$$

We obtain by direct calculation that

$$
E\left(u_{n}\right)=E\left(u_{n, 1}\right)+E\left(u_{n, 2}\right)+\rho_{n}
$$


where

$$
\begin{aligned}
\rho_{n}= & \frac{1}{2} \int_{\tilde{R}}^{\tilde{R}+a}\left[\left(\left|u_{n}^{\prime}\right|^{2}-\left|u_{n, 1}^{\prime}\right|^{2}-\left|u_{n, 2}^{\prime}\right|^{2}\right)-\frac{c}{x^{2}}\left(\left|u_{n}\right|^{2}-\left|u_{n, 1}\right|^{2}-\left|u_{n, 2}\right|^{2}\right)\right] d x \\
& -\frac{1}{p+1} \int_{\tilde{R}}^{\tilde{R}+a}\left(\left|u_{n}\right|^{p+1}-\left|u_{n, 1}\right|^{p+1}-\left|u_{n, 2}\right|^{p+1}\right) d x .
\end{aligned}
$$

We show that there exists $\tilde{R}>0$ and $a>1$, such that for $n$ large enough $\left|\rho_{n}\right| \leqslant(1 / 4-c) \frac{\delta}{4}$. First we observe by the properties of the cut-off that

$$
\left|\frac{1}{2} \int_{\tilde{R}}^{\tilde{R}+a}\left(\left|u_{n}^{\prime}\right|^{2}-\left|u_{n, 1}^{\prime}\right|^{2}-\left|u_{n, 2}^{\prime}\right|^{2}\right) d x\right| \leqslant \frac{5}{2} \int_{\tilde{R}}^{\tilde{R}+a}\left|u_{n}^{\prime}\right|^{2} d x+\frac{8}{a^{2}} \int_{\tilde{R}}^{\tilde{R}+a}\left|u_{n}\right|^{2} d x .
$$

We claim that there exist $\tilde{R}>0$ and $a>1$ such that for a subsequence $\left\{u_{n_{k}}\right\}$ we have

$$
\int_{\tilde{R}}^{\tilde{R}+a}\left|u_{n_{k}}^{\prime}\right|^{2} d x<\frac{1}{20}(1 / 4-c) \delta
$$

Suppose that this claim does not hold, that is for all $R>0, a>1$ there exists $k \in \mathbb{N}$ such that for all $n \geqslant k$ the following holds

$$
\int_{R}^{R+a}\left|u_{n}^{\prime}\right|^{2} d x \geqslant \frac{1}{20}(1 / 4-c) \delta .
$$

Let $\left(R_{1}, R_{1}+a_{1}\right)$. There exists $k_{1} \in \mathbb{N}$, such that for all $n \geqslant k_{1}$ we have

$$
\int_{R_{1}}^{R_{1}+a_{1}}\left|u_{n}^{\prime}\right|^{2} d x \geqslant \frac{1}{20}(1 / 4-c) \delta .
$$

Now let $R_{2}>R_{1}+a_{1}$ and $a_{2}>1$. Then by our assumption there exists $k_{2} \in \mathbb{N}$, such that for all $n \geqslant k_{2}$ it holds that

$$
\int_{R_{2}}^{R_{2}+a_{2}}\left|u_{n}^{\prime}\right|^{2} d x \geqslant \frac{1}{20}(1 / 4-c) \delta .
$$

Hence, there exists a subsequence $\left\{v_{n_{k}}\right\}_{k \in \mathbb{N}}$ such that for all $j \in\{1,2\}$ it holds that

$$
\int_{R_{j}}^{R_{j}+a_{j}}\left|u_{n_{k}}^{\prime}\right|^{2} d x \geqslant \frac{1}{20}(1 / 4-c) \delta
$$

for all $k \in \mathbb{N}$. Therefore, we can construct for all $l \in \mathbb{N}$ a subsequence $\left\{u_{n_{k}}\right\}_{k \in \mathbb{N}}$, such that for all $1 \leqslant j \leqslant l$ there are disjoint intervals $A_{j}=\left(R_{j}, R_{j}+a_{j}\right)$, such that

$$
\int_{A_{j}}\left|u_{n_{k}}^{\prime}\right|^{2} d x \geqslant \frac{1}{20}(1 / 4-c) \delta .
$$

Hence for all $l \in \mathbb{N}$ there exists a subsequence $\left\{u_{n_{k}}\right\}_{k \in \mathbb{N}}$, such that for all $k \in \mathbb{N}$ we have

$$
\int_{0}^{\infty}\left|u_{n_{k}}^{\prime}\right|^{2} d x \geqslant \sum_{j=1}^{l} \int_{A_{j}}\left|u_{n_{k}}^{\prime}\right|^{2} d x \geqslant \frac{l}{20}(1 / 4-c) \delta .
$$


This implies that $\int_{0}^{\infty}\left|u_{n_{k}}^{\prime}\right|^{2} d x \rightarrow \infty$, which is a contradiction since $\left\{u_{n}\right\}_{n \in \mathbb{N}}$ is bounded in $H_{0}^{1}\left(\mathbb{R}^{+}\right)$. Hence the assertion (3.10) is true. Now we note that

$$
\int_{0}^{R}\left|u_{n}\right|^{p+1} d x \leqslant\left\|u_{n}\right\|_{L^{\infty}}^{p-1} \int_{0}^{R}\left|u_{n}\right|^{2} d x .
$$

Since $\left\{u_{n}\right\}_{n \in \mathbb{N}}$ is bounded in $L^{\infty}\left(\mathbb{R}^{+}\right)$, in view of (3.6) we obtain for $R>0$ given in (3.6) that

$$
\int_{0}^{R}\left|u_{n}\right|^{2} d x \rightarrow 0 \quad \text { implies } \quad \int_{0}^{R}\left|u_{n}\right|^{p+1} d x \rightarrow 0 .
$$

For large $n$ we have $\tilde{R}+a<y_{n}-R$, since $y_{n} \rightarrow \infty$ by our assumption. Now (3.11) implies

$$
\begin{aligned}
& \left.\left|\frac{8}{a^{2}} \int_{\tilde{R}}^{\tilde{R}+a}\right| u_{n}\right|^{2} d x|+| \int_{\tilde{R}}^{\tilde{R}+a} \frac{c}{x^{2}}\left(\left|u_{n}\right|^{2}-\left|u_{n, 1}\right|^{2}-\left|u_{n, 2}\right|^{2}\right) d x \mid \\
& \quad+\left|\frac{1}{p+1} \int_{\tilde{R}}^{\tilde{R}+a}\left(\left|u_{n}\right|^{p+1}-\left|u_{n, 1}\right|^{p+1}-\left|u_{n, 2}\right|^{p+1}\right) d x\right| \\
& \leqslant\left.\left|\frac{8}{a^{2}} \int_{\tilde{R}}^{\tilde{R}+a}\right| u_{n}\right|^{2} d x\left|+\frac{c}{\tilde{R}^{2}}\right| \int_{\tilde{R}}^{\tilde{R}+a}\left|u_{n}\right|^{2}\left(1-\xi^{2}-(1-\xi)^{2}\right) d x \mid \\
& \quad+\left.\left|\frac{1}{p+1} \int_{\tilde{R}}^{\tilde{R}+a}\right| u_{n}\right|^{p+1}\left(1-\xi^{p+1}-(1-\xi)^{p+1}\right) d x \mid \\
& \leqslant
\end{aligned}
$$

for large $n$. Now (3.10) and (3.12) implies

$$
\left|\rho_{n}\right| \leqslant \frac{(1 / 4-c) \delta}{4} .
$$

Let us observe that $\left\|u_{n, 1}\right\|_{L^{p+1}} \rightarrow 0$ by (3.11). Hence

$$
E\left(u_{n, 1}\right)=\frac{1}{2} H\left(u_{n, 1}\right)+o(1) .
$$

Now let us notice that $\operatorname{supp}\left(u_{n, 2}\right) \subset(\tilde{R}, \infty)$. Moreover, in view of $(3.6)$,

$$
\int_{0}^{\infty}\left|u_{n, 2}\right|^{2} d x=\int_{y_{n}-R}^{\infty}\left|u_{n, 2}\right|^{2} d x+o(1) .
$$

Hence

$$
\int_{0}^{\infty} \frac{\left|u_{n, 2}\right|^{2}}{x^{2}} d x=\int_{y_{n}-R}^{\infty} \frac{\left|u_{n, 2}\right|^{2}}{x^{2}} d x+o(1) \leqslant \frac{\mu}{\left|y_{n}-R\right|^{2}} .
$$

Now $y_{n} \rightarrow \infty$ implies that

$$
E\left(u_{n, 2}\right)=E^{\infty}\left(u_{n, 2}\right)+o(1) .
$$

Thus,

$$
E\left(u_{n}\right)=\frac{1}{2} H\left(u_{n, 1}\right)+E^{\infty}\left(u_{n, 2}\right)+\rho_{n}+o(1) .
$$


From the properties of the cut-off and (3.6), we get

$$
\left\|u_{n, 2}\right\|_{L^{2}}^{2}=\left\|u_{n}\right\|_{L^{2}}^{2}-\left\|u_{n, 1}\right\|_{L^{2}}^{2}-2 \operatorname{Re} \int_{R^{\prime}}^{R^{\prime}+a} u_{n, 1} \bar{u}_{n, 2} d x \rightarrow \mu .
$$

Since $\frac{1}{2} H\left(u_{n, 1}\right)+\rho_{n}>0$ by (3.9) and (3.13), we obtain

$$
I=\lim _{n \rightarrow \infty} E\left(u_{n}\right) \geqslant \lim _{n \rightarrow \infty} E^{\infty}\left(u_{n, 2}\right) \geqslant I^{\infty} .
$$

which is a contradiction, hence (3.7) follows.

Now, from (3.7) we obtain

$$
\begin{gathered}
\lim _{n \rightarrow \infty}\left(\frac{1}{2} \int_{0}^{\infty}\left|u_{n}^{\prime}\right|^{2} d x-\frac{c}{2} \int_{0}^{\infty} \frac{\left|u_{n}\right|^{2}}{x^{2}} d x-\frac{1}{p+1} \int_{0}^{\infty}\left|u_{n}\right|^{p+1} d x\right)= \\
=\lim _{n \rightarrow \infty}\left(\frac{1}{2} \int_{0}^{\infty}\left|u_{n}^{\prime}\right|^{2} d x-\frac{1}{p+1} \int_{0}^{\infty}\left|u_{n}\right|^{p+1} d x\right) .
\end{gathered}
$$

Hence

$$
I \geqslant I^{\infty},
$$

which is again a contradiction. Thus $\left\{y_{n}\right\}_{n \in \mathbb{N}}$ is bounded and has an accumulation point $y^{*} \in \mathbb{R}^{+}$. Therefore, it follows that for any $\varepsilon>0$ there is $R>0$ such that

$$
\int_{0}^{R}\left|u_{n}\right|^{2} \geqslant \mu-\varepsilon
$$

for all $n \in \mathbb{N}$. Hence $u_{n} \rightarrow u$ strongly in $L^{2}\left(\mathbb{R}^{+}\right)$. Moreover, since $\left\{u_{n}\right\}$ is bounded in $H_{0}^{1}\left(\mathbb{R}^{+}\right)$it is also strongly convergent in $L^{p+1}\left(\mathbb{R}^{+}\right)$. By the weaklower semicontinuity of $H$ (see [17]), it follows that $E(u) \leqslant \lim _{n \rightarrow \infty} E\left(u_{n}\right)=I$. Hence $E(u)=I$, and $E\left(u_{n}\right) \rightarrow E(u)$ implies that $H\left(u_{n}\right) \rightarrow H(u)$, which concludes that proof.

Remark 3.4. If $c<0$, the infimum is not attained on the $L^{2}$ constraint. Indeed, let us assume that there exists $v \in H_{0}^{1}\left(\mathbb{R}^{+}\right)$, such that $\|v\|_{L^{2}}^{2}=\mu$ and $E(v)=I$. Then taking translates of $v$, i.e. $v(\cdot-y)$ for $y>0$, we get $E(v(\cdot-y))<I$, which is a contradiction.

Lemma 3.5. Let $0<c<1 / 4, \omega>0$ and $1<p<5$. Let $\mu$ be defined by Lemma 2.10. Then $u \in H_{0}^{1}\left(\mathbb{R}^{+}\right)$is a ground state solution of (2.1) if and only if $u$ solves the minimization problem

$$
\left\{\begin{array}{l}
u \in \Gamma \\
S(u)=\inf \{S(v): v \in \Gamma\}
\end{array}\right.
$$

Proof. Step 1. Let us first define

$$
m_{\mathcal{A}}=\inf \{S(u): u \in \mathcal{A}\},
$$

and

$$
m_{\Gamma}=\inf \{S(u): u \in \Gamma\} .
$$

If $u \in \mathcal{G}$, then $S(u)=m_{\Gamma}$. By Lemma 2.10 we know that $u \in \Gamma$, hence $m_{\mathcal{A}} \leqslant m_{\Gamma}$. 
Step 2. We claim that every solution of (3.14) belongs to $\mathcal{A}$. Indeed, let us consider a solution $u$ to (3.14). There exists a Lagrange multiplier $\lambda_{1} \in \mathbb{R}$ such that $S^{\prime}(u)=\lambda_{1} u$. Hence there exists $\lambda \in \mathbb{R}$ such that

$$
-u^{\prime \prime}-\frac{c}{x^{2}} u+\lambda \omega u=|u|^{p-1} u \text {. }
$$

Indeed, since $u$ is a solution of (3.14), and for $\lambda>0$ let

$$
u_{\lambda}(x)=\lambda^{1 / 2} u(\lambda x) .
$$

We have $u_{\lambda} \in \Gamma$. Since $u_{1}$ is a solution of (3.14), we get from (3.15) and Lemma 2.2 that

$$
\left.\frac{\partial}{\partial \lambda} S\left(u_{\lambda}\right)\right|_{\lambda=1}=\left\|u^{\prime}\right\|_{L^{2}}^{2}-c\left\|\frac{u}{x}\right\|_{L^{2}}^{2}-\frac{p-1}{2(p+1)}\|u\|_{L^{p+1}}^{p+1}=0 .
$$

We can deduce directly from (3.15) and (3.16) that

$$
\lambda \omega \mu=\frac{p+3}{p-1} H(u),
$$

which implies that $\lambda>0$. Let us define $v$ by

$$
u(x)=\lambda^{1 /(p-1)} v\left(\lambda^{1 / 2} x\right) .
$$

By (3.16), $v \in \mathcal{A}$, hence

$$
S(v) \geqslant m_{\mathcal{A}} .
$$

We obtain simple calculation that

$$
m_{\Gamma}=S(u)=\lambda^{2 /(p-1)+1 / 2} S(v)+(1-\lambda) \frac{\omega \mu}{2} .
$$

Hence,

$$
m_{\mathcal{A}} \geqslant \lambda^{\frac{2}{p-1}+\frac{1}{2}} m_{\mathcal{A}}+(1-\lambda) \frac{\omega \mu}{2} .
$$

Since $u$ is a solution of (3.15), we obtain from Lemma 2.2 that $m_{\mathcal{A}} \geqslant 0$. By Lemma 2.2 and Lemma 2.10 we have that

$$
\frac{\omega \mu}{2}=\left(\frac{2}{p-1}+\frac{1}{2}\right) m_{\mathcal{A}}
$$

hence

$$
0 \geqslant \lambda^{\frac{2}{p-1}+\frac{1}{2}}-\lambda\left(\frac{2}{p-1}+\frac{1}{2}\right)+\left(\frac{2}{p-1}-\frac{3}{2}\right) .
$$

The right hand side is always strictly positive, except if $\lambda=1$. Thus, $\lambda=1$, which implies together with (3.16) that $u \in \mathcal{A}$.

Step 3. It follows from Step 2, that $m_{\Gamma} \leqslant m_{\mathcal{A}}$, hence $m_{\Gamma}=m_{\mathcal{A}}$. In particular, it follows that if $u \in \mathcal{G}$, then $u \in \Gamma$ and $S(u)=m_{\mathcal{A}}$, thus $u$ satisfies (3.14). Conversely, let $u$ be the solution of (3.14). Then by Step $2 u \in \mathcal{A}$, and $S(u)=$ $m_{\Gamma}=m_{\mathcal{A}}$, hence $u \in \mathcal{G}$. 
Theorem 3.6. Let $0<c<1 / 4, \omega>0$, and $1<p<5$. If $\varphi$ is a ground state solution of (2.1), then the standing wave $u(t, x)=e^{i \omega t} \varphi(x)$ is an orbitally stable solution of (1.1), i.e. for all $\varepsilon>0$ there is $\delta>0$, such that if $u(0) \in H_{0}^{1}\left(\mathbb{R}^{+}\right)$ satisfies $\|\varphi-u(0)\|_{H^{1}}<\delta$, then the corresponding maximal solution $u$ of $(1.1)$ satisfies

$$
\sup _{t \in \mathbb{R}} \inf _{\theta \in \mathbb{R}}\left\|u(t)-e^{i \theta} \varphi\right\|_{H^{1}}<\varepsilon
$$

Proof. Assume by contradiction that there exist a sequence $\left\{\varphi_{n}\right\}_{n \in \mathbb{N}} \subset H_{0}^{1}\left(\mathbb{R}^{+}\right)$, a sequence $\left\{t_{n}\right\}_{n \in \mathbb{N}} \subset \mathbb{R}$, and $\varepsilon>0$, such that

$$
\lim _{n \rightarrow \infty}\left\|\varphi_{n}-\varphi\right\|_{H^{1}}=0
$$

and the corresponding maximal solution $u_{n}$ of (1.1) with initial value $\varphi_{n}$ satisfies

$$
\inf _{\theta \in \mathbb{R}}\left\|u_{n}\left(t_{n}\right)-e^{i \theta} \varphi\right\|_{H^{1}} \geqslant \varepsilon .
$$

Set $v_{n}=u_{n}\left(t_{n}\right)$. Applying Lemma 3.5, we obtain

$$
\lim _{n \rightarrow \infty} \inf _{\varphi \in \mathcal{G}}\left\|v_{n}-\varphi\right\|_{H^{1}} \geqslant \varepsilon \text {. }
$$

By the conservation of charge and energy, we obtain

$$
\left\|v_{n}\right\|_{L^{2}}^{2} \rightarrow \mu \text {, and } E\left(v_{n}\right) \rightarrow I \text {. }
$$

Hence $\left\{v_{n}\right\}_{n \in \mathbb{N}}$ is a minimizing sequence of (3.1). It follows from Lemma 3.3, that there exists a solution $u$ of the problem (3.1), such that $\left\|v_{n}-u\right\|_{H^{1}} \rightarrow 0$. By Lemma 3.5 we obtain that $u \in \mathcal{G}$, which contradicts (3.17).

\section{Instability}

In this section we assume that $p \geqslant 5$. Let us define for $v \in H_{0}^{1}\left(\mathbb{R}^{+}\right)$the functional

$$
Q(v)=\left\|v^{\prime}\right\|_{L^{2}}^{2}-c\left\|\frac{v}{x}\right\|_{L^{2}}^{2}-\frac{p-1}{2(p+1)}\|v\|_{L^{p+1}}^{p+1} .
$$

In Lemma 2.2 we have shown that if $v$ is a solution of $(2.1)$, then $Q(v)=0$. First, we prove the virial identities.

Proposition 4.1. Let $u_{0} \in H_{0}^{1}\left(\mathbb{R}^{+}\right)$be such that $x u_{0} \in L^{2}\left(\mathbb{R}^{+}\right)$and $u$ be the corresponding maximal solution to (1.1). Then $x u(t) \in L^{2}\left(\mathbb{R}^{+}\right)$for any $t \in$ $\left(-T_{\min }, T_{\max }\right)$. Moreover, the following identities hold for all $v \in H_{0}^{1}\left(\mathbb{R}^{+}\right)$:

$$
\begin{aligned}
\frac{\partial}{\partial t}\|x u(t)\|_{L^{2}}^{2} & =4 \operatorname{Im} \int_{0}^{\infty} \bar{u}(t) x u^{\prime}(t) d x, \\
\frac{\partial^{2}}{\partial t^{2}}\|x u(t)\|_{L^{2}}^{2} & =8 Q(u(t)) .
\end{aligned}
$$

Proof. The proof follows the same line as in [6]. 
Proposition 4.2. Let $p \geqslant 5$ and let $u_{0} \in H_{0}^{1}\left(\mathbb{R}^{+}\right)$be such that

$$
x u_{0} \in L^{2}\left(\mathbb{R}^{+}\right) \text {and } E\left(u_{0}\right)<0 .
$$

Then the maximal solution $u$ to (1.1) with initial condition $u_{0}$ blows up in finite time.

Proof. First, let us note that

$$
Q(u(t))=2 E(u(t))+\frac{5-p}{2(p+1)}\|u(t)\|_{L^{p+1}}^{p+1} .
$$

Since $p \geqslant 5$, we get by the conservation of the energy that

$$
Q(u(t)) \leqslant 2 E\left(u_{0}\right)<0 \text { for all } t \in\left(-T_{\min }, T_{\max }\right) .
$$

Hence, Proposition 4.1 implies that

$$
\frac{\partial^{2}}{\partial t^{2}}\|x u(t)\|_{L^{2}}^{2} \leqslant 16 E\left(u_{0}\right) \text { for all } t \in\left(-T_{\min }, T_{\max }\right) .
$$

Integrating twice, we get

$$
\|x u(t)\|_{L^{2}}^{2} \leqslant 8 E\left(u_{0}\right) t^{2}+\left(4 \operatorname{Im} \int_{0}^{\infty} \bar{u}_{0} x u_{0}^{\prime} d x\right) t+\left\|x u_{0}\right\|_{L^{2}}^{2}
$$

The main coefficient of the second order polynomial on the right hand side is negative. Thus, it is negative for $|t|$ large, what contradicts with $\|x u(t)\|_{L^{2}}^{2} \geqslant 0$ for all $t$. Therefore, $-T_{\min }>-\infty$ and $T_{\max }<+\infty$.

Theorem 4.3. Assume that $\omega>0$ and $p=5$. Then for any solution $\varphi \in$ $H_{0}^{1}\left(\mathbb{R}^{+}\right)$of $(2.1)$ the standing wave $e^{i \omega t} \varphi(x)$ is unstable by blow-up.

Proof. Since $p=5$, we have for all $v \in H_{0}^{1}\left(\mathbb{R}^{+}\right)$, that $2 E(v)=Q(v)$. Hence from Lemma 2.2 we get that

$$
E(\varphi)=0
$$

Let us define $\varphi_{n, 0}=\left(1+\frac{1}{n}\right) \varphi$. It is easy to see that $E\left(\varphi_{n, 0}\right)<0$. By Lemma 2.1 we know that $x \varphi_{n, 0} \in L^{2}\left(\mathbb{R}^{+}\right)$. The conclusion follows from Proposition 4.2.

Theorem 4.4. Let $p>5$. Then for any ground state solution $\varphi$ to (2.1), the corresponding standing wave $e^{i \omega t} \varphi(x)$ is orbitally unstable.

We need to prove a series of Lemmas to establish Theorem 4.4.

Lemma 4.5. Let $v \in H_{0}^{1}\left(\mathbb{R}^{+}\right) \backslash\{0\}$ such that $Q(v) \leqslant 0$, and set $v_{\lambda}(x)=$ $\lambda^{1 / 2} v(\lambda x)$ for $\lambda>0$. Then there exists $\lambda^{*} \in(0,1]$ such that the following assertions hold:

(1) $Q\left(v_{\lambda^{*}}\right)=0$.

(2) $\lambda^{*}=1$ if and only if $Q(v)=0$.

(3) $\frac{\partial}{\partial \lambda} S\left(v_{\lambda}\right)=\frac{1}{\lambda} Q\left(v_{\lambda}\right)$.

(4) $\frac{\partial}{\partial \lambda} S\left(v_{\lambda}\right)>0$ for all $\lambda \in\left(0, \lambda^{*}\right)$, and $\frac{\partial}{\partial \lambda} S\left(v_{\lambda}\right)<0$ for all $\lambda \in\left(\lambda^{*},+\infty\right)$.

(5) The function $\left(\lambda^{*},+\infty\right) \ni \lambda \mapsto S\left(v_{\lambda}\right)$ is concave. 
Proof. We get that by the scaling properties of $\lambda \mapsto Q\left(v_{\lambda}\right)$ that

$$
Q\left(v_{\lambda}\right)=\lambda^{2}\left\|v^{\prime}\right\|_{L^{2}}^{2}-\lambda^{2} c\left\|\frac{v}{x}\right\|_{L^{2}}^{2}-\lambda^{\frac{p-1}{2}} \frac{p-1}{2(p+1)}\|v\|_{L^{p+1}}^{p+1} .
$$

We get from the Hardy inequality that for $c \in(0,1 / 4)$

$$
\begin{aligned}
& (1-4 c) \lambda^{2}\left\|v^{\prime}\right\|_{L^{2}}^{2}-\lambda^{\frac{p-1}{2}} \frac{p-1}{2(p+1)}\|v\|_{L^{p+1}}^{p+1} \\
& \quad \leqslant Q\left(v_{\lambda}\right) \leqslant \lambda^{2}\left\|v^{\prime}\right\|_{L^{2}}^{2}-\lambda^{\frac{p-1}{2}} \frac{p-1}{2(p+1)}\|v\|_{L^{p+1}}^{p+1} .
\end{aligned}
$$

Since $p>5$, there exists $\lambda \in(0,1]$ small enough, such that $Q\left(v_{\lambda}\right)>0$. Hence, there exists $\lambda^{*} \in(0,1]$, such that $Q\left(v_{\lambda^{*}}\right)=0$. This proves (1). To prove (2), we first note that if $\lambda^{*}=1$, then clearly $Q(v)=0$. Now assume that $Q(v)=0$. Then

$$
\begin{aligned}
Q\left(v_{\lambda}\right) & =\lambda^{2} Q(v)+\left(\lambda^{2}-\lambda^{\frac{p-1}{2}}\right) \frac{p-1}{2(p+1)}\|v\|_{L^{p+1}}^{p+1} \\
& =\left(\lambda^{2}-\lambda^{\frac{p-1}{2}}\right) \frac{p-1}{2(p+1)}\|v\|_{L^{p+1}}^{p+1},
\end{aligned}
$$

which is positive for all $\lambda \in(0,1)$, since $p>5$. Hence, (2) follows. (3) follows form simple calculation:

$$
\begin{aligned}
\frac{\partial}{\partial \lambda} S\left(v_{\lambda}\right) & =\lambda\left\|v^{\prime}\right\|_{L^{2}}^{2}-\lambda c\left\|\frac{v}{x}\right\|_{L^{2}}^{2}-\lambda^{\frac{p-1}{2}-1} \frac{p-1}{2(p+1)}\|v\|_{L^{p+1}}^{p+1} \\
& =\frac{1}{\lambda} Q\left(v_{\lambda}\right) .
\end{aligned}
$$

To show (4), we note that

$$
Q\left(v_{\lambda}\right)=\frac{\lambda^{2}}{\left(\lambda^{*}\right)^{2}} Q\left(v_{\lambda^{*}}\right)+\lambda^{2}\left(\left(\lambda^{*}\right)^{\frac{p-5}{2}}-\lambda^{\frac{p-5}{2}}\right) \frac{p-1}{2(p+1)}\|v\|_{L^{p+1}}^{p+1} .
$$

Since $p>5$ and $Q\left(v_{\lambda^{*}}\right)=0$, we get that $\lambda>\lambda^{*}$ implies $Q\left(v_{\lambda}\right)<0$, and $\lambda<\lambda^{*}$ implies $Q\left(v_{\lambda}\right)>0$. This and (3), implies (4).

Finally, we get by simple calculation that

$$
\frac{\partial^{2}}{\partial \lambda^{2}} S\left(v_{\lambda}\right)=\frac{1}{\lambda^{2}} Q\left(v_{\lambda}\right)-\lambda^{\frac{p-5}{2}}\left(\frac{p-1}{2}-2\right) \frac{p-1}{2(p+1)}\|v\|_{L^{p+1}}^{p+1} .
$$

Since $p>5$, we obtain for $\lambda>\lambda^{*}$ that $\frac{\partial^{2}}{\partial \lambda^{2}} S\left(v_{\lambda}\right)<0$ which concludes the proof of (5).

To prove orbital instability we prove a new variational characterization of the ground state. Let us define the following set

$$
\mathcal{M}=\left\{v \in H_{0}^{1}\left(\mathbb{R}^{+}\right) \backslash\{0\}: Q(v)=0, J(v) \leqslant 0\right\},
$$

and the corresponding minimization problem

$$
d=\inf _{W \in \mathcal{M}} S(W) .
$$

Then we have the following. 
Lemma 4.6. The following equality holds:

$$
m=d
$$

where $m$ is defined by (2.4).

Proof. Let $v \in \mathcal{G}$. Since $v$ solves (2.1), by Lemma 2.2 we have that $Q(v)=$ $J(v)=0$, hence $\mathcal{G} \subset \mathcal{M}$, and

$$
d \leqslant m .
$$

Let now $v \in \mathcal{M}$. Assume first, that $J(v)=0$. In this case $v \in \mathcal{N}$, and $m \leqslant S(v)$. Let us assume that $J(v)<0$. Then for $v_{\lambda}(x)=\lambda^{1 / 2} v(\lambda x)$ we have

$$
J\left(v_{\lambda}\right)=\lambda^{2}\left\|v^{\prime}\right\|_{L^{2}}^{2}-\lambda^{2} c\left\|\frac{v}{x}\right\|_{L^{2}}^{2}+\omega\|v\|_{L^{2}}^{2}-\lambda^{(p-1) / 2}\|v\|_{L^{p+1}}^{p+1},
$$

and $\lim _{\lambda \downarrow 0} J\left(v_{\lambda}\right)>\omega\|v\|_{L^{2}}^{2}$, thus there exists $\lambda_{1} \in(0,1)$, such that $J\left(v_{\lambda_{1}}\right)=0$. By Proposition 2.9

$$
m \leqslant S\left(v_{\lambda_{1}}\right) .
$$

From $Q(v)=0$ and Lemma 4.5 we have

$$
S\left(v_{\lambda_{1}}\right) \leqslant S(v)
$$

hence $m \leqslant S(v)$ for all $v \in \mathcal{M}$. Therefore $m \leqslant d$, which concludes the proof.

We now define the manifold

$$
\mathcal{J}=\left\{u \in H_{0}^{1}\left(\mathbb{R}^{+}\right) \backslash\{0\}: J(u)<0, Q(u)<0, S(u)<d\right\} .
$$

We will prove the invariance of $\mathcal{J}$ under the flow of (1.1).

Lemma 4.7. Let $u_{0} \in \mathcal{J}$ and $u \in C\left(\left(-T_{\min }, T_{\max }\right), H_{0}^{1}\left(\mathbb{R}^{+}\right)\right)$the corresponding solution to (1.1). Then $u(t) \in \mathcal{J}$ for all $t \in\left(-T_{\min }, T_{\max }\right)$.

Proof. Let $u_{0} \in \mathcal{J}$ and $u \in C\left(\left(-T_{\min }, T_{\max }\right), H_{0}^{1}\left(\mathbb{R}^{+}\right)\right)$the corresponding maximal solution. Since $S$ is conserved under the flow of (1.1) we have for all $t \in\left(-T_{\min }, T_{\max }\right)$ that

$$
S(u(t))=S\left(u_{0}\right)<d .
$$

We prove the assertion by contradiction. Suppose that there exists $t \in\left(-T_{\min }, T_{\max }\right)$ such that

$$
J(u(t)) \geqslant 0 .
$$

Then, since $J$ and $u$ are continuous, there exists $t_{0} \in\left(-T_{\min }, T_{\max }\right)$ such that

$$
J\left(u\left(t_{0}\right)\right)=0,
$$

thus $u\left(t_{0}\right) \in \mathcal{N}$. Then by Proposition 2.9 we have that

$$
S\left(u\left(t_{0}\right)\right) \geqslant d,
$$

which is a contradiction, thus $J(u(t))<0$ for all $t \in\left(-T_{\min }, T_{\max }\right)$. Let us suppose now that for some $t \in\left(-T_{\min }, T_{\max }\right)$ we have

$$
Q(u(t)) \geqslant 0 \text {. }
$$


Again, by continuity, there exists $t_{1} \in\left(-T_{\min }, T_{\max }\right)$ such that

$$
Q\left(u\left(t_{1}\right)\right)=0 .
$$

Hence we that $Q\left(u\left(t_{1}\right)\right)=0$, and $J\left(u\left(t_{1}\right)\right)<0$. Therefore, by Lemma 4.6

$$
S\left(u\left(t_{1}\right)\right) \geqslant d,
$$

which is a contradiction. Hence,

$$
Q(u(t))<0
$$

for all $t \in\left(-T_{\min }, T_{\max }\right)$, which concludes the proof.

Lemma 4.8. Let $u_{0} \in \mathcal{J}$ and $u \in C\left(\left(-T_{\min }, T_{\max }\right), H_{0}^{1}\left(\mathbb{R}^{+}\right)\right)$. Then there exists $\varepsilon>0$ such that $Q(u(t)) \leqslant-\varepsilon$ for all $t \in\left(-T_{\min }, T_{\max }\right)$.

Proof. Let $u_{0} \in \mathcal{J}$ and let us define $v:=u(t)$ and $v_{\lambda}(x)=\lambda^{1 / 2} v(\lambda x)$. By Lemma 4.5 , there exists $\lambda_{0}<1$ such that $Q\left(v_{\lambda^{*}}\right)=0$. If $J\left(v_{\lambda^{*}}\right) \leqslant 0$, then by Lemma 4.7 we get $S\left(v_{\lambda^{*}}\right) \geqslant m$. On the other hand, if $J\left(v_{\lambda^{*}}\right)>0$, there exists $\lambda_{1} \in\left(\lambda^{*}, 1\right)$, such that $J\left(\lambda_{1}\right)=0$ and we replace $\lambda^{*}$ with $\lambda_{1}$. In this case, by Lemma 4.6 we get $S\left(v_{\lambda^{*}}\right) \geqslant m$. In conclusion, in both cases we obtain

$$
S\left(v_{\lambda^{*}}\right) \geqslant d \text {. }
$$

By Lemma 4.5 we know that $\lambda \mapsto S\left(v_{\lambda}\right)$ is concave on $\left(\lambda^{*},+\infty\right)$, thus

$$
S(v)-S\left(v_{\lambda^{*}}\right) \geqslant\left.\left(1-\lambda^{*}\right) \frac{\partial}{\partial \lambda} S\left(v_{\lambda}\right)\right|_{\lambda=1} .
$$

From Lemma 4.5 we have

$$
\left.\frac{\partial}{\partial \lambda} S\left(v_{\lambda}\right)\right|_{\lambda=1}=Q(v)
$$

Moreover, since $Q(v)<0$ and $\lambda^{*} \in(0,1)$, we have

$$
\left(1-\lambda^{*}\right) Q(v)>Q(v) .
$$

Combining (4.2)-(4.5), we obtain

$$
S(v)-d>Q(v) .
$$

Define $-\varepsilon=S(v)-d$. Then $\varepsilon>0$, since $v \in \mathcal{J}$. Owing to the conservation of the energy and mass, $\varepsilon>0$ is independent from $t$, which concludes the proof.

Lemma 4.9. Let us take $u_{0} \in \mathcal{J}$ such that $x u_{0} \in L^{2}\left(\mathbb{R}^{+}\right)$. Then the maximal solution $u \in C\left(\left(-T_{\min }, T_{\max }\right), H_{0}^{1}\left(\mathbb{R}^{+}\right)\right)$corresponding to the initial value problem (1.1) blows up in finite time.

Proof. From Lemma 4.8 we know that there exists $\varepsilon>0$ such that

$$
Q(u(t))<-\varepsilon \text { for } t \in\left(-T_{\min }, T_{\max }\right) .
$$

From Proposition 4.1 we know that $\frac{\partial^{2}}{\partial t^{2}}\|x u(t)\|_{L^{2}}^{2}=8 Q(u(t))$, and by integration we get

$$
\|x u(t)\|_{L^{2}}^{2} \leqslant-4 \varepsilon t^{2}+C_{1} t+C_{2} .
$$


The right hand side of (4.6) is negative for large $|t|$, which contradicts with $\|x u(t)\|_{L^{2}}^{2}>0$ for all $t$. Therefore, $T_{\min }>-\infty$ and $T_{\max }<\infty$ and by local well-posedness it follows that

$$
\lim _{t \downarrow-T_{\min }}\|u(t)\|_{H^{1}}=+\infty, \text { and } \lim _{t \uparrow T_{\max }}\|u(t)\|_{H^{1}}=+\infty .
$$

Proof of Theorem 4.4. Let $\varphi \in \mathcal{G}$. Owing to Lemma 4.9, it suffices to show that there exists a sequence $\left\{\varphi_{\lambda}\right\} \subset \mathcal{J}$, which converges to $\varphi$ in $H_{0}^{1}\left(\mathbb{R}^{+}\right)$. Let us put $\varphi_{\lambda}(x)=\lambda^{1 / 2} \varphi(\lambda x)$. By Lemma $4.5\left\{\varphi_{\lambda}\right\} \subset \mathcal{J}$ for all $\lambda \in(0,1)$. Additionally, by Proposition 2.1, $\varphi$ decays exponentially at infinity, and so does $\varphi_{\lambda}$. Therefore, $x \varphi_{\lambda} \in L^{2}\left(\mathbb{R}^{+}\right)$. Clearly, $\varphi_{\lambda} \rightarrow \varphi$ as $\lambda \rightarrow 0$, and by Lemma 4.9 the maximal solution of (1.1) corresponding to $\varphi_{\lambda}$, blows up in finite time for all $\lambda \in(0,1)$. Hence, the conclusion follows.

Funding Open Access funding enabled and organized by Projekt DEAL.

Open Access. This article is licensed under a Creative Commons Attribution 4.0 International License, which permits use, sharing, adaptation, distribution and reproduction in any medium or format, as long as you give appropriate credit to the original author(s) and the source, provide a link to the Creative Commons licence, and indicate if changes were made. The images or other third party material in this article are included in the article's Creative Commons licence, unless indicated otherwise in a credit line to the material. If material is not included in the article's Creative Commons licence and your intended use is not permitted by statutory regulation or exceeds the permitted use, you will need to obtain permission directly from the copyright holder. To view a copy of this licence, visit http://creativecommons. org/licenses/by/4.0/.

Publisher's Note Springer Nature remains neutral with regard to jurisdictional claims in published maps and institutional affiliations. 


\section{Appendix}

We prove the following Lemma:

Lemma 5.1. Let $\psi_{A}(x)=q(x+A)-q(x-A)$, where $q$ is (2.6). Then $\psi_{A} \in$ $H_{0}^{1}\left(\mathbb{R}^{+}\right)$and for large $A>0$, we have the following approximations:

$$
\begin{aligned}
\int_{0}^{\infty}\left|\psi_{A}^{\prime}\right|^{2} d x & =\int_{-\infty}^{\infty}\left|q^{\prime}\right|^{2} d x+O\left(\left(2 A+\frac{1}{\sqrt{\omega}}\right) e^{-2 \sqrt{\omega} A}\right), \\
\int_{0}^{\infty}\left|\psi_{A}\right|^{2} d x & =\int_{-\infty}^{\infty}|q|^{2} d x+O\left(\left(2 A+\frac{1}{\sqrt{\omega}}\right) e^{-2 \sqrt{\omega} A}\right), \\
\int_{0}^{\infty} \frac{\left|\psi_{A}(x)\right|^{2}}{x^{2}} & \lesssim \frac{1}{A^{2}} \int_{-\infty}^{\infty}|q|^{2} d x+O\left(\frac{1}{A^{2}} e^{-\sqrt{\omega} A}\right), \\
\int_{0}^{\infty}\left|\psi_{A}(x)\right|^{p+1} d x & =\int_{-\infty}^{\infty}|q|^{p+1} d x+O\left(e^{-2 \sqrt{\omega} A}\right) .
\end{aligned}
$$

Proof. We will use the fact that $q(x) \leqslant M e^{-\sqrt{\omega}|x|}$ and $q^{\prime}(x) \leqslant M e^{-\sqrt{\omega}|x|}$ for some $M>0$.

We get (5.1) by using the symmetry of $q$ and $q^{\prime}$ :

$$
\int_{0}^{\infty}\left|\psi_{A}^{\prime}\right|^{2} d x=\int_{-\infty}^{\infty}\left|q^{\prime}\right|^{2} d x-\int_{-\infty}^{\infty} q^{\prime}(x+A) q^{\prime}(x-A) d x .
$$

We estimate the second term by

$$
\begin{aligned}
& \left|\int_{-\infty}^{\infty} q^{\prime}(x+A) q^{\prime}(x-A) d x\right| \\
& \quad \lesssim \int_{-\infty}^{\infty} e^{-\sqrt{\omega}|x+A|-\sqrt{\omega}|x-A|} d x=\left(\left(2 A+\frac{1}{\sqrt{\omega}}\right) e^{-2 \sqrt{\omega} A}\right)
\end{aligned}
$$

hence (5.1) follows. We get (5.2) the same way.

We now show (5.3). From Hardy's inequality we get

$$
\int_{0}^{A / 2} \frac{\left|\psi_{A}\right|^{2}}{x^{2}} d x \leqslant 4 \int_{0}^{A / 2}\left|\psi_{A}^{\prime}(x)\right|^{2}=O\left(e^{-\sqrt{\omega} A}\right) .
$$

Moreover, we have

$$
\int_{A / 2}^{\infty} \frac{\left|\psi_{A}(x)\right|^{2}}{x^{2}} d x \leqslant \frac{4}{A^{2}} \int_{A / 2}^{\infty}\left|\psi_{A}\right|^{2} d x=\frac{4}{A^{2}} \int_{-\infty}^{\infty}|q|^{2}+O\left(\frac{1}{A^{2}} e^{-\sqrt{\omega} A}\right) .
$$

Hence

$$
\begin{aligned}
\int_{0}^{\infty} \frac{\left|\psi_{A}\right|^{2}}{x^{2}} d x & =\int_{0}^{A / 2} \frac{\left|\psi_{A}\right|^{2}}{x^{2}} d x+\int_{A / 2}^{\infty} \frac{\left|\psi_{A}\right|^{2}}{x^{2}} d x \\
& \leqslant \frac{4}{A^{2}} \int_{-\infty}^{\infty}|q|^{2} d x+O\left(\frac{1}{A^{2}} e^{-\sqrt{\omega} A}\right),
\end{aligned}
$$

which is the estimate in (5.3).

To show (5.4), we use the fact that

$|q(x-A)-q(x+A)|^{p+1}=q^{p+1}(x-A)-(p+1) q^{p}(x-A) q(x+A)+O\left(q^{2}(x+A)\right)$. 
We get

$$
\begin{aligned}
\int_{0}^{\infty} q^{p+1}(x-A) d x & =\int_{-\infty}^{\infty} q^{p+1}(x) d x-\int_{-\infty}^{-A} q^{p+1}(x) d x \\
& =\int_{-\infty}^{\infty} q^{p+1}(x) d x+O\left(e^{-\sqrt{\omega}(p+1) A}\right) \\
\int_{0}^{\infty} q^{p}(x-A) q(x+A) d x & \lesssim \int_{0}^{\infty} e^{-\sqrt{\omega} p|x-A|-\sqrt{\omega}|x+A|} d x=O\left(e^{-2 \sqrt{\omega} A}\right), \\
\int_{0}^{\infty} O\left(q^{2}(x+A)\right) d x & =O\left(e^{-2 \sqrt{\omega} A}\right) .
\end{aligned}
$$

Hence

$$
\int_{0}^{\infty}\left|\psi_{A}(x)\right|^{p+1} d x=\int_{-\infty}^{\infty}|q|^{p+1} d x+O\left(e^{-2 \sqrt{\omega} A}\right) .
$$

This concludes the proof.

We now state the proof of Lemma 2.8. The proof follows the arguments of the paper [11], with some important modifications. We introduce the norm

$$
\|u\|^{2}=\int_{0}^{\infty}\left(\left|u^{\prime}\right|^{2}-c \frac{|u|^{2}}{x^{2}}+\omega|u|^{2}\right) d x,
$$

which is equivalent to the standard norm on $H_{0}^{1}\left(\mathbb{R}^{+}\right)$if $0<c<1 / 4$.

Proof of Lemma 2.8. Step 1. There exists $u_{0} \in H_{0}^{1}\left(\mathbb{R}^{+}\right)$, such that, up to a subsequence, $u_{n}$ is weakly convergent to $u_{0}$ in $H_{0}^{1}\left(\mathbb{R}^{+}\right)$, and $S^{\prime}\left(u_{0}\right)=0$.

Since $\left\{u_{n}\right\}_{n \in \mathbb{N}}$ is bounded in $H_{0}^{1}\left(\mathbb{R}^{+}\right)$, it admits a weakly convergent subsequence in $H_{0}^{1}\left(\mathbb{R}^{+}\right)$with a weak limit $u_{0} \in H_{0}^{1}\left(\mathbb{R}^{+}\right)$. We only need to show that $S^{\prime}\left(u_{0}\right)=0$. Since by our assumption $S^{\prime}\left(u_{n}\right) \rightarrow 0$, it suffices to show that for all $\varphi \in C_{0}^{\infty}\left(\mathbb{R}^{+}\right)$we have

$$
S^{\prime}\left(u_{n}\right) \varphi-S^{\prime}\left(u_{0}\right) \varphi \rightarrow 0
$$

Indeed, we have

$$
\begin{aligned}
S^{\prime}\left(u_{n}\right) \varphi-S^{\prime}\left(u_{0}\right) \varphi= & \operatorname{Re} \int_{0}^{\infty}\left(u_{n}^{\prime}-u_{0}^{\prime}\right) \bar{\varphi}^{\prime} d x-c \operatorname{Re} \int_{0}^{\infty} \frac{\left(u_{n}-u_{0}\right) \bar{\varphi}}{x^{2}} d x \\
& +\omega \operatorname{Re} \int_{0}^{\infty}\left(u_{n}-u_{0}\right) \bar{\varphi} d x \\
& -\operatorname{Re} \int_{0}^{\infty}\left(\left|u_{n}\right|^{p-1} u_{n}-\left|u_{0}\right|^{p-1} u_{0}\right) \bar{\varphi} d x .
\end{aligned}
$$

Since $u_{n} \rightarrow u_{0}$ in $H_{0}^{1}\left(\mathbb{R}^{+}\right)$and strongly in $L_{\text {loc }}^{q}\left(\mathbb{R}^{+}\right)$for all $q \geqslant 1$, our statement follows.

Let us set $v_{n}=u_{n}-u_{0}$.

Step 2. Assume that

$$
\sup _{z \in \mathbb{R}^{+}} \int_{B_{1}(z)}\left|v_{n}\right|^{2} d x \rightarrow 0,
$$

where $B_{1}(z)$ is the unit ball centered at $z$. Then $u_{n} \rightarrow u_{0}$ strongly in $H_{0}^{1}\left(\mathbb{R}^{+}\right)$, and Lemma 2.8 holds with $k=0$. 
Using the fact that $S^{\prime}\left(u_{0}\right)=0$, we get

$$
\begin{aligned}
S^{\prime}\left(u_{n}\right) v_{n}= & \operatorname{Re} \int_{0}^{\infty} u_{n}^{\prime} \bar{v}_{n}^{\prime} d x-c \operatorname{Re} \int_{0}^{\infty} \frac{u_{n} \bar{v}_{n}}{x^{2}} d x+\omega \operatorname{Re} \int_{0}^{\infty} u_{n} \bar{v}_{n} d x \\
& -\operatorname{Re} \int_{0}^{\infty}\left|u_{n}\right|^{p-1} u_{n} \bar{v}_{n} d x= \\
= & \left\|v_{n}\right\|^{2}+\operatorname{Re} \int_{0}^{\infty}\left(\left|u_{0}\right|^{p-1} u_{0}-\left|u_{n}\right|^{p-1} u_{n}\right) \bar{v}_{n} d x .
\end{aligned}
$$

Hence,

$$
\left\|v_{n}\right\|^{2}=S^{\prime}\left(u_{n}\right) v_{n}+\operatorname{Re} \int_{0}^{\infty}\left(\left|u_{n}\right|^{p-1} u_{n}-\left|u_{0}\right|^{p-1} u_{0}\right) \bar{v}_{n} d x .
$$

We recall that $S^{\prime}\left(u_{n}\right) \rightarrow 0$. Hölder's inequality implies that

$$
\left.\left|\int_{0}^{\infty}\right| u_{n}\right|^{p-1} u_{n} v_{n} d x \mid \leqslant\left\|u_{n}\right\|_{L^{p+1}}^{p}\left\|v_{n}\right\|_{L^{p+1}} .
$$

Assumption (5.5) and Lemma 1.1 in [16] implies that $\left\|v_{n}\right\|_{L^{p+1}} \rightarrow 0$. Hence

$$
\operatorname{Re} \int_{0}^{\infty}\left|u_{n}\right|^{p-1} u_{n} \bar{v}_{n} d x \rightarrow 0 .
$$

We obtain similarly that $\operatorname{Re} \int_{0}^{\infty}\left|u_{0}\right|^{p-1} u_{0} \bar{v}_{n} d x \rightarrow 0$, hence $\left\|v_{n}\right\|^{2} \rightarrow 0$, which completes the proof of Step 2.

Step 3. Assume that there exist $\left\{z_{n}\right\}_{n \in \mathbb{N}} \subset \mathbb{R}^{+}$and $d>0$, such that

$$
\int_{B_{1}\left(z_{n}\right)}\left|v_{n}\right|^{2} d x \rightarrow d
$$

Then, up to a subsequence, we have for $q \in H^{1}(\mathbb{R})$, that (i) $z_{n} \rightarrow \infty$, (ii) $u_{n}\left(\cdot+z_{n}\right) \rightarrow q \neq 0$ in $H^{1}(\mathbb{R})$, and (iii) $S^{\infty^{\prime}}(q)=0$.

To show $(i)$, let us assume by contradiction that $\left\{z_{n}\right\}_{n \in \mathbb{N}}$ has an accumulation point $z^{*} \in \mathbb{R}^{+}$. Then for a subsequence of $\left\{v_{n}\right\}_{n \in \mathbb{N}}$ we have

$$
\int_{B_{2}\left(z^{*}\right)}\left|v_{n}\right|^{2} d x \geqslant d
$$

Since $v_{n} \rightarrow 0$ in $H_{0}^{1}\left(\mathbb{R}^{+}\right)$, we have $v_{n} \rightarrow 0$ in $L^{2}\left(B_{2}\left(z^{*}\right)\right)$, which implies that

$$
d \leqslant \lim _{n \rightarrow \infty} \int_{B_{2}\left(z^{*}\right)}\left|v_{n}\right|^{2} d x=0
$$

which is a contradiction, hence $(i)$ holds.

Since $u_{n}\left(\cdot+z_{n}\right)$ is bounded in $H^{1}(\mathbb{R})$ the re exists $q \in H^{1}(\mathbb{R})$ such that $u_{n}\left(\cdot+z_{n}\right)$ converges weakly to $q$ in $H^{1}(\mathbb{R})$. We only need to show that $q \neq 0$. Since $u_{0}\left(\cdot+z_{n}\right) \rightarrow 0$ in $H^{1}(\mathbb{R})$, we have that $v_{n}\left(\cdot+z_{n}\right) \rightarrow q$ in $H^{1}(\mathbb{R})$, and in $L_{\text {loc }}^{2}(\mathbb{R})$ in particular. Hence

$$
\int_{B_{1}(0)}|q(x)|^{2} d x=\lim _{n \rightarrow \infty} \int_{B_{1}(0)}\left|v_{n}\left(x+z_{n}\right)\right|^{2} d x=\int_{B_{1}\left(z_{n}\right)}\left|v_{n}(y)\right|^{2} d y \geqslant d>0 .
$$

This implies that $q \neq 0$. 
We finally show (iii). We define $\tilde{u}(\cdot)=u_{n}\left(\cdot+z_{n}\right)$. We obtain, similarly as in Step 1, that for any $\varphi \in C_{0}^{\infty}(\mathbb{R})$,

$$
S^{\infty \prime}\left(\tilde{u}_{n}\right) \varphi-S^{\infty \prime}(q) \varphi \rightarrow 0 .
$$

It remains to show that $S^{\infty^{\prime}}\left(\tilde{u}_{n}\right) \varphi \rightarrow 0$. For any fixed $\varphi \in C_{0}^{\infty}(\mathbb{R}), \varphi\left(\cdot-z_{n}\right)$ is in $H_{0}^{1}\left(\mathbb{R}^{+}\right)$for sufficiently big $n \in \mathbb{N}$. Hence, we obtain

$$
\begin{aligned}
S^{\prime}\left(u_{n}\right) \varphi\left(\cdot-z_{n}\right)= & \operatorname{Re} \int_{-z_{n}}^{\infty} u_{n}^{\prime}\left(x+z_{n}\right) \bar{\varphi}_{n}^{\prime}(x) d x-c \operatorname{Re} \int_{-z_{n}}^{\infty} \frac{u_{n}\left(x+z_{n}\right) \bar{\varphi}(x)}{\left(x+z_{n}\right)^{2}} d x \\
& +\omega \operatorname{Re} \int_{-z_{n}}^{\infty} u_{n}\left(x+z_{n}\right) \bar{\varphi}(x) d x \\
& -\operatorname{Re} \int_{-z_{n}}^{\infty}\left|u_{n}\left(x+z_{n}\right)\right|^{p-1} u_{n}\left(x+z_{n}\right) \bar{\varphi}(x) d x .
\end{aligned}
$$

Since $S^{\prime}\left(u_{n}\right) \rightarrow 0$ and $\varphi\left(\cdot-z_{n}\right)$ is bounded in $H^{1}(\mathbb{R})$, it follows

$$
\begin{aligned}
& \operatorname{Re} \int_{-z_{n}}^{\infty} \tilde{u}_{n}^{\prime}(x) \bar{\varphi}_{n}^{\prime}(x) d x-c \operatorname{Re} \int_{-z_{n}}^{\infty} \frac{\tilde{u}_{n}(x) \bar{\varphi}(x)}{\left(x+z_{n}\right)^{2}} d x \\
& \quad+\omega \operatorname{Re} \int_{-z_{n}}^{\infty} \tilde{u}_{n}(x) \bar{\varphi}(x) d x-\operatorname{Re} \int_{-z_{n}}^{\infty}\left|\tilde{u}_{n}(x)\right|^{p-1} \tilde{u}_{n}(x) \bar{\varphi}(x) d x \rightarrow 0 .
\end{aligned}
$$

Moreover, since $u_{n}$ is bounded in $L^{\infty}$, and $\varphi$ is compactly supported, we get

$$
\begin{aligned}
& \left|\operatorname{Re} \int_{-z_{n}}^{\infty} \frac{\tilde{u}_{n}(x) \bar{\varphi}(x)}{\left(x+z_{n}\right)^{2}} d x\right| \\
& \quad=\left|\operatorname{Re} \int_{0}^{\infty} \frac{u_{n}(x) \bar{\varphi}\left(x-z_{n}\right)}{x^{2}} d x\right| \leqslant \frac{1}{\left(z_{n}-\inf \{\operatorname{supp}(\varphi)\}\right)^{2}}\left\|u_{n} \varphi\right\|_{L^{\infty}} \rightarrow 0,
\end{aligned}
$$

Thus

$$
\begin{aligned}
S^{\infty \prime}\left(\tilde{u}_{n}\right) \varphi= & \operatorname{Re} \int_{-\infty}^{\infty} \tilde{u}_{n}^{\prime}(x) \bar{\varphi}_{n}^{\prime}(x) d x+\omega \operatorname{Re} \int_{-\infty}^{\infty} \tilde{u}_{n}(x) \bar{\varphi}(x) d x \\
& -\operatorname{Re} \int_{-\infty}^{\infty}\left|\tilde{u}_{n}(x)\right|^{p-1} \tilde{u}_{n}(x) \bar{\varphi}(x) d x \rightarrow 0
\end{aligned}
$$

which concludes the proof of Step 3.

Step 4. Suppose there exist $k \geqslant 1,\left\{x_{n}^{i}\right\} \subset \mathbb{R}^{+}, q_{i} \in H^{1}(\mathbb{R})$ for $1 \leqslant i \leqslant k$, such that

$$
\begin{array}{r}
x_{n}^{i} \rightarrow \infty, \quad\left|x_{n}^{i}-x_{n}^{j}\right| \rightarrow \infty \text { if } i \neq j, \\
u_{n}\left(\cdot+x_{n}^{i}\right) \rightarrow q_{i} \neq 0, \text { for all } 1 \leqslant i \leqslant k, \\
S^{\infty^{\prime}}\left(q_{i}\right)=0 .
\end{array}
$$

Then

(1) If $\sup _{z \in \mathbb{R}^{+}} \int_{B_{1}(z)}\left|u_{n}-u_{0}-\sum_{i=1}^{k} q_{i}\left(\cdot-x_{n}^{i}\right)\right|^{2} d x \rightarrow 0$ then

$$
\left\|u_{n}-u_{0}-\sum_{i=1}^{k} q_{i}\left(\cdot-x_{n}^{i}\right)\right\|_{H^{1}} \rightarrow 0 .
$$


(2) If there exist $\left\{z_{n}\right\} \subset \mathbb{R}^{+}$and $d>0$, such that

$$
\int_{B_{1}\left(z_{n}\right)}\left|u_{n}-u_{0}-\sum_{i=1}^{k} q_{i}\left(\cdot-x_{n}^{i}\right)\right|^{2} d x \rightarrow d,
$$

then, up to a subsequence, it follows that

$$
\text { (i) } z_{n} \rightarrow \infty \text {, and }\left|z_{n}-x_{n}^{i}\right| \rightarrow \infty \text { for all } 1 \leqslant i \leqslant k \text {, }
$$

$$
\text { (ii) } u_{n}\left(\cdot+z_{n}\right) \rightarrow q_{i+1} \quad \text { (iii) } S^{\infty \prime}\left(q_{i+1}\right)=0 .
$$

Suppose assumption (1) holds. We introduce $\xi_{n}=u_{n}-u_{0}-\sum_{i=1}^{k} q_{i}^{a}(\cdot-$ $\left.x_{n}^{i}\right)$, where $q_{i}^{a}$ is a suitable cut-off of $q_{i}$, such that $\operatorname{supp}\left(q_{i}^{a}\right) \subset(0, \infty)$. This is possible owing to the exponential decay of $q_{i}$ at infinity, and $x_{n}^{i} \rightarrow \infty$ as $n \rightarrow \infty$ for all $i$. We get

$$
\begin{aligned}
S^{\prime}\left(u_{n}\right) \xi_{n}= & \operatorname{Re} \int_{0}^{\infty} u_{n}^{\prime} \bar{\xi}_{n}^{\prime} d x-c \operatorname{Re} \int_{0}^{\infty} \frac{u_{n} \bar{\xi}_{n}}{x^{2}} d x+\omega \operatorname{Re} \int_{0}^{\infty} u_{n} \bar{\xi}_{n} d x \\
& -\operatorname{Re} \int_{0}^{\infty}\left|u_{n}\right|^{p-1} u_{n} \bar{\xi}_{n} d x \\
= & \left\|\xi_{n}\right\|^{2}+\operatorname{Re} \int_{0}^{\infty}\left(u_{0}^{\prime}+\sum_{i=1}^{k} q_{i}^{a \prime}\left(\cdot-x_{n}^{i}\right)\right) \bar{\xi}_{n}^{\prime} d x \\
& +\operatorname{Re} \int_{0}^{\infty}\left(\omega-\frac{c}{x^{2}}\right)\left(u_{0}+\sum_{i=1}^{k} q_{i}^{a}\left(\cdot-x_{n}^{i}\right)\right) \bar{\xi}_{n} d x \\
& -\operatorname{Re} \int_{0}^{\infty}\left|u_{n}\right|^{p-1} u_{n} \bar{\xi}_{n} d x .
\end{aligned}
$$

Since $S^{\prime}\left(u_{0}\right) \xi_{n}=0$, we get

$$
\begin{aligned}
S^{\prime}\left(u_{n}\right) \xi_{n}= & \left\|\xi_{n}\right\|^{2}+\operatorname{Re} \int_{0}^{\infty}\left(\left|u_{0}\right|^{p-1} u_{0}-\left|u_{n}\right|^{p-1} u_{n}\right) \bar{\xi}_{n} d x \\
& +\operatorname{Re} \int_{0}^{\infty} \sum_{i=1}^{k} q_{i}^{a \prime}\left(\cdot-x_{n}^{i}\right) \bar{\xi}_{n}^{\prime} d x \\
& +\operatorname{Re} \int_{0}^{\infty}\left(\omega-\frac{c}{x^{2}}\right) \sum_{i=1}^{k} q_{i}^{a}\left(\cdot-x_{n}^{i}\right) \bar{\xi}_{n} d x
\end{aligned}
$$

Using the fact that $\left\|\xi_{n}\right\|_{L^{p+1}} \rightarrow 0$ by Lemma 1.1 in [16], we get that the second term of the right hand side converges to zero. Now, from the weak convergence of $\xi_{n}$ to zero and that $S^{\prime}\left(u_{n}\right) \rightarrow 0$,we obtain that $\left\|\xi_{n}\right\| \rightarrow 0$.

Suppose now that assumption (2) holds. Then (i) and (ii) follows as in Step 3. To show (ii), let us set $\tilde{u}_{n}=u_{n}\left(\cdot+z_{n}\right)$. We note that

$$
S^{\infty^{\prime}}\left(\tilde{u}_{n}\right) \varphi-S^{\infty^{\prime}}(q) \varphi \rightarrow 0,
$$

for all $\varphi \in C_{0}^{\infty}(\mathbb{R})$. Now $S^{\infty^{\prime}}\left(\tilde{u}_{n}\right) \rightarrow 0$ follows similarly as in Step 3, which concludes the proof.

Step 5. Conclusion By Step 1 we know that $u_{n} \rightarrow u_{0}$ and $S^{\prime}\left(u_{0}\right)=0$. Hence (i) of Lemma 2.8 is verified. If the assumption of Step 2 holds, then Lemma 2.8 
is true with $k=0$. Otherwise, the assumption of Step 3 holds. We have to iterate Step 4 . We only need to show that assumption 1 of Step 4 occurs after a finite number of iterations. Let us notice that

$$
\begin{aligned}
& \left\|u_{n}-u_{0}-\sum_{i=1}^{k} q_{i}\left(\cdot-x_{i}^{n}\right)\right\|_{H^{1}}^{2} \\
& \quad=\left\|u_{n}\right\|_{H^{1}}^{2}+\left\|u_{0}\right\|_{H^{1}}^{2}+\sum_{i=1}^{k}\left\|q_{i}\right\|_{H^{1}}^{2}-2\left\langle u_{n}, u_{0}+\sum_{i=1}^{k} q_{i}\left(\cdot-x_{i}^{n}\right)\right\rangle_{H^{1}} .
\end{aligned}
$$

Moreover, since $u_{n} \rightarrow u_{0}$ and $u_{n}\left(\cdot+x_{i}^{n}\right) \rightarrow q_{i}$, we get for the last term that

$$
\left\langle u_{n}, u_{0}+\sum_{i=1}^{k} q_{i}\left(\cdot-x_{i}^{n}\right)\right\rangle_{H^{1}} \rightarrow\left\|u_{0}\right\|_{H^{1}}^{2}+\sum_{i=1}^{k}\left\|q_{i}\right\|_{H^{1}}^{2},
$$

Now since $u_{n}$ converges weakly to $u_{0}$, we obtain for $k \geqslant 1$ that

$\lim _{n \rightarrow \infty}\left\|u_{n}\right\|_{H^{1}}^{2}-\left\|u_{0}\right\|_{H^{1}}^{2}-\sum_{i=1}^{k}\left\|q_{i}\right\|_{H^{1}}^{2}=\lim _{n \rightarrow \infty}\left\|u_{n}-u_{0}-\sum_{i=1}^{k} q_{i}\left(\cdot-x_{i}^{n}\right)\right\|_{H^{1}}^{2} \geqslant 0$.

Since $q_{i}$ is a nontrivial critical point of $S^{\infty}$, it is true that $\left\|q_{i}\right\|_{H^{1}} \geqslant \epsilon>0$. Hence, after a finite number of iterations assumption 1 of Step 4 must occur.

Finally, we have to verify that

$$
S\left(u_{n}\right) \rightarrow S\left(u_{0}\right)+\sum_{i=1}^{k} S^{\infty}\left(q_{i}\right)
$$

We first show that

$$
S\left(u_{n}\right) \rightarrow S\left(u_{0}\right)+S^{\infty}\left(v_{n}\right) .
$$

A straightforward calculation gives

$$
\begin{aligned}
S\left(u_{n}\right)= & S\left(u_{0}\right)+S^{\infty}\left(v_{n}\right)+\operatorname{Re} \int_{0}^{\infty} u_{0}^{\prime}\left(\bar{u}_{n}^{\prime}-\bar{u}_{0}^{\prime}\right) d x-c \operatorname{Re} \int_{0}^{\infty} \frac{u_{0}\left(\bar{u}_{n}-\bar{u}_{0}\right)}{x^{2}} d x \\
& +\omega \operatorname{Re} \int_{0}^{\infty} u_{0}\left(\bar{u}_{n}-\bar{u}_{0}\right) d x-\frac{c}{2} \int_{0}^{\infty} \frac{\left|u_{n}-u_{0}\right|^{2}}{x^{2}} d x \\
& +\frac{1}{p+1}\left(\left\|u_{n}-u_{0}\right\|_{L^{p+1}}^{p+1}-\left\|u_{n}\right\|_{L^{p+1}}^{p+1}+\left\|u_{n}\right\|_{L^{p+1}}^{p+1}\right)
\end{aligned}
$$

From a lemma by Brezis and Lieb (see e.g. Lemme 4.6 [12]) we have

$$
\int_{0}^{\infty}\left|u_{n}-u_{0}\right|^{p+1} d x-\int_{0}^{\infty}\left|u_{n}\right|^{p+1} d x+\int_{0}^{\infty}\left|u_{0}\right|^{p+1} d x \rightarrow 0 .
$$

Hence (5.7) follows. It only remains to show that

$$
S^{\infty}\left(v_{n}\right) \rightarrow \sum_{i=1}^{k} S^{\infty}\left(q_{i}\right)
$$


We calculate

$$
\begin{aligned}
S\left(v_{n}\right)= & \frac{1}{2}\left\|v_{n}-\sum_{i=1}^{k} q_{i}\left(\cdot-x_{i}^{n}\right)\right\|_{H^{1}}^{2}+\frac{1}{2}\left\|\sum_{i=1}^{k} q_{i}\left(\cdot-x_{i}^{n}\right)\right\|_{H^{1}}^{2} \\
& +\left\langle v_{n}-\sum_{i=1}^{k} q_{i}\left(\cdot-x_{i}^{n}\right), \sum_{i=1}^{k} q_{i}\left(\cdot-x_{i}^{n}\right)\right\rangle_{H^{1}}-\frac{1}{p+1}\left\|\sum_{i=1}^{k} q_{i}\left(\cdot-x_{i}^{n}\right)\right\|_{L^{p+1}}^{p+1} \\
& -\frac{1}{p+1}\left\|v_{n}\right\|_{L^{p+1}}^{p+1}+\frac{1}{p+1}\left\|\sum_{i=1}^{k} q_{i}\left(\cdot-x_{i}^{n}\right)\right\|_{L^{p+1}}^{p+1} \cdot
\end{aligned}
$$

We have shown that $v_{n}-\sum_{i=1}^{k} q_{i}\left(\cdot-x_{i}^{n}\right) \rightarrow 0$ strongly in $H^{1}$. Hence the first and third term above converges to zero as $n \rightarrow \infty$. By using Sobolev's inequality and $\|A-B\| \geqslant|\|A\|-\|B\||$ we have

$$
\left\|\sum_{i=1}^{k} q_{i}\left(\cdot-x_{i}^{n}\right)\right\|_{L^{p+1}}^{p+1}-\left\|v_{n}\right\|_{L^{p+1}}^{p+1} \rightarrow 0,
$$

which concludes the proof.

\section{References}

[1] Bensouilah, A., Dinh, V.D., Zhu, S.: On stability and instability of standing waves for the nonlinear Schrödinger equation with inverse-square potential. J. Math. Phys. 59, (2018)

[2] Bruneau, L., Dereziński, J., Georgescu, V.: Homogeneous Schrödinger operators on half-line. Annales Henri Poincare 12(3), 547-590 (2009)

[3] Cazenave, T.: Semilinear Schrödinger Equations. American Mathematical Society, Providence (2003)

[4] Cazenave, T., Lions, P.L.: Orbital stability of standing waves for some nonlinear Schrödinger equations. Commun. Math. Phys. 85, 549-561 (1982)

[5] Cazenave, T., Haraux, A.: An Introduction to Semilinear Evolution Equations. Oxford Lecture Series in Mathematics and its Applications 13 (1998)

[6] Csobo, E., Genoud, F.: Minimal mass blow-up solutions for the $L^{2}$ critical NLS with inverse-square potential. Nonlinear Anal. 168, 110-129 (2018)

[7] Davies, E.B.: A review of Hardy inequalities. Operator Theory Adv. Appl. 110(2), 55-68 (1999)

[8] Dinh, V.D.: Global existence and blow-up for a class of the focusing nonlinear Schrödinger equation with inverse-square potential. J. Math. Anal. Appl. 468(1), 270-303 (2018) 
[9] Fukuizumi, R., Jeanjean, L.: Stability for standing waves for a nonlinear Schrödinger equation with repulsive Dirac delta potentials. Discrete Continuous Dyn. Syst. 21(1), 121-136 (2008)

[10] Hajaiej, H., Stuart, C.A.: On the variational approach to the stability of standing waves of the nonlinear Schrödinger equation. Adv. Nonlinear Stud. 469-501 (2004)

[11] Jeanjean, L., Tanaka, K.: A positive solution for a nonlinear Schrödinger equation on $\mathbb{R}^{N}$. Indiana Univ. Math. J. 443-464 (2005)

[12] Kavian, O.: Introduction á la Thèorie des Points Critiques et Applications aux Problèmes Elliptiques. Springer, Berlin (1993)

[13] Kovarik, H., Truc, F.: Schrödinger operators on a half-line with inverse square potentials. Math. Model. Nat. Phenomena 170-176 (2014)

[14] Le Coz, S.: Standing waves in nonlinear Schrödinger equations, Analytical and numerical aspects of partial. Differ. Equ. 151-192 (2009)

[15] Lions, P.L.: The concentration-compactness principle in the calculus of variations. The limit case, part 1, Annales de L. H. P., section C, 109-145 (1984)

[16] Lions, P.L.: The concentration-compactness principle in the calculus of variations. The locally compact case, part 2. Annales de L. H. P., section C, 223-283 (1984)

[17] Montefusco, E.: Lower semicontinuity of functionals via the concentrationcompactness principle. J. Math. Anal. Appl. 263, 264-276 (2001)

[18] Okazawa, N., Suzuki, T., Yokota, T.: Energy methods for abstract nonlinear Schrödinger equations. Evol. Equ. Control Theory 1, 337-354 (2012)

[19] Shioji, N., Watanabe, K.: A generalized Pohozaev identity and uniqueness of positive radial solutions of $\Delta u+g(r) u+h(r) u^{p}=0$. J. Differ. Equ. 255, 44484475 (2013)

[20] Struwe, M.: Variational Methods, 2nd edn. Springer, Berlin (1991)

[21] Suzuki, T.: Nonlinear Schrödinger equations with inverse square potentials in two dimensional space. Differ. Equ. Appl. Dyn. Syst. 1019-1024 (2015)

[22] Trachanas, G.P., Zographopoulos, N.B.: Orbital stability for the Schrödinger operator involving inverse square potential. J. Differ. Equ. 259, 4989-5016 (2015)

[23] Weinstein, M.I.: Nonlinear Schrödinger equations and sharp interpolation estimates. Commun. Math. Phys. 87, 567-576 (1982) 
Elek Csobo

Institut für Mathematik

Goethe-Universität Frankfurt

Robert-Mayer-Str. 10

60629 Frankfurt am Main

Germany

e-mail: Csobo@math.uni-frankfurt.de

Received: 10 February 2021.

Accepted: 21 June 2021. 